\title{
CONSTITUINTES QUÍMICOS DE Capraria biflora (Scrophulariaceae) E ATIVIDADE LARVICIDA DE SEU ÓLEO ESSENCIAL ${ }^{\#}$
}

\author{
Luciana Gregório da S. Souza, Macia Cleane S. Almeida, Francisco José Q. Monte, Gilvandete Maria P. Santiago, \\ Raimundo Braz-Filho"\# e Telma Leda G. Lemos* \\ Departamento de Química Orgânica e Inorgânica, Universidade Federal do Ceará, Campus do Pici, 60451-970 Fortaleza - CE, Brasil \\ Clerton L. Gomes e Ronaldo F. do Nascimento \\ Departamento de Química Analítica e Físico-Química, Centro de Cências, Universidade Federal do Ceará, Campus do Pici, \\ 60451-970 Fortaleza - CE, Brasil
}

Recebido em 8/3/12; aceito em 28/6/12; publicado na web em 5/10/12

\begin{abstract}
CHEMICAL CONSTITUENTS OF Capraria biflora (SCROPHULARIACEAE) AND LARVICIDAL ACTIVITY OF ESSENTIAL OIL. Analysis of essential oil from fresh leaves of Capraria biflora allowed identification of fourteen essential oil constituents among which thirteen are sesquiterpene compounds, and $\alpha$-humulene $(43.0 \%)$ the major constituent. The essential oil was tested for larvicidal activity against Aedes aegypti showing good activity, with $\mathrm{LC}_{50} 73.39 \mu \mathrm{g} / \mathrm{mL}(2.27 \mathrm{~g} / \mathrm{mL})$. Chromatographic studies of extracts from roots and stems allowed the isolation of five compounds: naphthoquinone biflorin, sesquiterpene caprariolide B, the steroid $\beta$-sitosterol, the carbohydrate D-mannitol and iridoid myopochlorin first reported in the species $C$. biflora. The structures of compounds were characterized by spectroscopic data, IR, MS, NMR ${ }^{13} \mathrm{C}, \mathrm{NMR}^{1} \mathrm{H}, \mathrm{NOE}, \mathrm{HSQC}$ and HMBC.
\end{abstract}

Keywords: chlorinated iridoid; Capraria biflora; essential oil.

\section{INTRODUÇÃO}

A família Scrophulariaceae possui distribuição cosmopolita, apresentando cerca de 270 gêneros e aproximadamente 5100 espécies. ${ }^{1} \mathrm{~A}$ espécie Capraria biflora é originária das Antilhas e América do Sul e habita zonas temperadas ou áreas de clima tropical. ${ }^{2,3}$ Trata-se de uma planta invasora, sendo encontrada em terrenos baldios, preferindo locais úmidos como riachos ou lagoas. No Brasil, é encontrada principalmente em Goiás, Minas Gerais e na faixa litorânea que se estende do Piauí ate o Espírito Santo. ${ }^{4}$

Esta espécie é conhecida popularmente como chá-da-terra, chá-do-méxico, chá-da-martinica, chá-de-goteira, chá-de-pé-da-calçada, chá-do-rio, chá-da-américa, chá-das-antilhas, chá-de-lima, chá-de-marajó, chá-da-balsaminha, chá-do-maranhão, chá-bravo e chá-de-preto. ${ }^{5}$ Dentre os usos descritos na literatura para esta espécie, destacam-se: tratamento de dor, febre, gripe, vômitos, hemorroidas, reumatismo e como antitumoral. ${ }^{6-8}$ Estudos farmacológicos realizados também demonstraram potencial citotóxico, analgésico, anti-inflamatório e antibiótico. ${ }^{8}$ Estudos fitoquímicos realizados com um espécime desta espécie relataram a presença de diversas classes de substâncias, destacando-se as naftoquinonas biflorina e seu dímero bis-biflorina, ${ }^{2,9}$ os sesquiterpenos caprariolídeos $\mathrm{A}-\mathrm{D}^{10}$ e os iridoides harpagida e 8-O-benzoilarpagida.,

Dando continuidade ao estudo de plantas medicinais do Nordeste brasileiro, este trabalho relata os resultados obtidos de uma reinvestigação fitoquímica das raízes e do caule, incluindo-se a extração do óleo essencial das folhas, que foi testado como agente larvicida sobre Aedes aegypti. Vale ressaltar que, apesar desta espécie já ter sido objeto de estudos fitoquímicos, sua reinvestigação revelou resultados adicionais também importantes, já que permitiu o isolamento da orto-quinona biflorina e da miopoclorina, substância ainda não

*e-mail: tlemos@dqoi.ufc.br

\#Artigo em homenagem ao Prof. Otto R. Gottlieb (31/8/1920-19/6/2011)

\#\# Pesquisador Visitante Emérito - FAPERJ/UENF/UFRRJ descrita nesta espécie, além da extração do óleo essencial utilizado na realização de testes biológicos importantes.

\section{PARTE EXPERIMENTAL}

\section{Procedimentos experimentais gerais}

Os pontos de fusão foram determinados em equipamentos de micro determinação Mettler provido de placa aquecedora FP-52 e unidade de controle FP-5. As determinações foram realizadas a uma velocidade de aquecimento de $4{ }^{\circ} \mathrm{C} / \mathrm{min}$ e os valores obtidos não foram corrigidos.

Os espectros de absorção na região do Infravermelho (IV) foram obtidos em espectrômetro Perkin Elmer FT-IR Spectrum 1000, com transformada de Fourier, utilizando-se pastilha de KBr.

Os espectros de ressonância magnética nuclear de hidrogênio $\left(\mathrm{RMN}^{1} \mathrm{H}\right)$ e carbono $\left(\mathrm{RMN}^{13} \mathrm{C}\right)$ foram obtidos em espectrômetro Brucker, modelo DRX- 500 operando nas frequências do hidrogênio e do carbono a 500,13 e 125,75 MHz, respectivamente.

A análise qualitativa do óleo essencial foi realizada em CG-EM da marca Shimadzu, modelo GCMS-QP2010 Plus. As análises foram realizadas em coluna capilar de sílica fundida DB-5 (30 m x 0,25 mm e espessura de filme $0,25 \mu \mathrm{m}$ ), sendo utilizado hélio (He) como gás de arraste, em fluxo de $1,33 \mathrm{~mL} / \mathrm{min}$. A injeção em modo split 1:20 foi realizada com injetor a $220^{\circ} \mathrm{C}$. A temperatura da fonte de íons foi de $240{ }^{\circ} \mathrm{C}$ e da interface $260^{\circ} \mathrm{C}$. O forno foi programado para uma temperatura de $60^{\circ} \mathrm{C}$ (isotérmica por $1 \mathrm{~min}$ ), com posterior incremento de $3^{\circ} \mathrm{C} / \mathrm{min}$ até $240^{\circ} \mathrm{C}$. As concentrações dos compostos foram calculadas a partir das áreas dos picos do cromatograma e foram dispostas por ordem de eluição (Tabela 1).

A quantificação dos constituintes do óleo essencial foi realizada em cromatógrafo da marca Shimadzu, modelo GC-17A-FID. Os espectros de massas foram obtidos com a voltagem de ionização de $70 \mathrm{eV}$. As análises foram realizadas em coluna capilar de sílica fundida DB-5 (30 m x 0,25 mm e espessura de filme $0,25 \mu \mathrm{m}$ ), sendo utilizado hidrogênio $(\mathrm{H})$ como gás de arraste, em fluxo de 
0,8 mL/min. A injeção em modo split 1:20 foi realizada com injetor a $200{ }^{\circ} \mathrm{C}$. A temperatura do detector foi de $230^{\circ} \mathrm{C}$ e o forno foi programado para uma temperatura de $60^{\circ} \mathrm{C}$ (isotérmica por $1 \mathrm{~min}$ ), com posteriores incrementos de $10^{\circ} \mathrm{C} / \mathrm{min}$ até $120^{\circ} \mathrm{C}$ e $3^{\circ} \mathrm{C} / \mathrm{min}$ até 180 ${ }^{\circ} \mathrm{C}$. Os constituintes foram identificados através dos índices de Kovats corrigidos por regressão linear, calculados em relação aos tempos de retenção de uma série homóloga de n-alcanos e comparação de seus espectros de massa com aqueles do banco de dados NIST05, bem como comparação visual com espectros de massas registrados na literatura. ${ }^{11}$

Os espectros de massas de alta resolução foram obtidos em espectrômetro Shimadzu, modelo LCMS-IT-TOF, equipado com fonte de ionização por electrospray.

A determinação de rotação óptica foi realizada em um polarímetro 341 da Perkim Elmer.

Nas colunas cromatográficas foi utilizado gel de sílica 60 (Ø 63 - $200 \mu \mathrm{m}$ ) como fase estacionária. O comprimento e diâmetro das colunas variaram de acordo com as massas das amostras, bem como as quantidades de sílica a serem utilizadas. Para cromatografia em camada delgada (CCD), utilizou-se gel de sílica 60 (Ø 2 - $25 \mu \mathrm{m})$ sobre placas de vidro e sobre alumínio com indicador de fluorescência na faixa de $254 \mathrm{~nm}$. A revelação das substâncias nas cromatoplacas foi realizada pela pulverização com solução (1:1) de vanilina a $1 \%$ em etanol com solução de ácido perclórico a 5\% seguido de aquecimento.

\section{Material vegetal}

As raízes e caule de $C$. biflora foram coletados em maio de 2008 no município de Itapiúna, CE. Posteriormente, as folhas foram coletadas em março de 2011 também em Itapiúna, CE. A espécie foi identificada pelo Botânico E. Nunes e as exsicatas encontram-se depositadas no Herbário Prisco Bezerra do Departamento de Biologia da Universidade Federal do Ceará com os números 30.848 e 30.849.

\section{Extração dos constituintes voláteis}

A extração de óleo essencial das folhas de C. biflora foi realizada pelo método de hidrodestilação, utilizando doseador tipo Cleavenger.

Folhas frescas de C. biflora $(654,0 \mathrm{~g})$ foram acondicionadas em balão de 5,0 L, juntamente com 1,5 L de água destilada. $\mathrm{O}$ material foi mantido em ebulição por $2 \mathrm{~h}$, obtendo-se um óleo de coloração amarelada $(0,059 \%)$. A fase aquosa (hidrolato) foi reservada para posterior análise de atividade larvicida. O óleo essencial obtido das folhas de $C$. biflora foi analisado por CG-EM e CG-DIC.

\section{Isolamento dos constituintes fixos}

As raízes $(700,0 \mathrm{~g})$ de $C$. biflora secas e trituradas foram submetidas à extração com éter de petróleo à temperatura ambiente $\mathrm{e}$ na ausência de luz. O solvente foi evaporado sob pressão reduzida, obtendo-se o respectivo extrato (EEPR, 1,7 g). O extrato EEPR (1,0 g) foi adsorvido em gel de sílica $(15,0 \mathrm{~g})$ e submetido à coluna cromatográfica, utilizando-se como eluentes hexano, AcOEt e $\mathrm{MeOH}$, puros e em misturas binárias, em ordem crescente de polaridade. Foram coletadas 14 frações com volume médio de $200 \mathrm{~mL}$. As frações 8-10 (hexano/AcOEt 8/2, v/v) foram reunidas após comparação em cromatografia em camada delgada (CCD), obtendo-se um composto de coloração violeta, a orto-naftoquinona biflorina 1 (58 mg). A fração 2-5 (hexano/AcOEt 9/1, v/v ) (600 mg) foi submetida a sucessivas cromatografias em coluna, levando ao isolamento do sesquiterpeno caprariolídeo B 2 (21 mg) e do esteroide $\beta$-sitosterol (15 mg).

O extrato do caule previamente obtido por extração com etanol, do material triturado, (CBEC, 60,0 g) foi submetido à cromatografia em gel de sílica (30,0 g) sob pressão reduzida, tendo como eluentes hexano, AcOEt e $\mathrm{MeOH}$ puros, e forneceu as frações denominadas de $\mathrm{EHC}(3,5 \mathrm{~g}), \mathrm{EAC}(8,0 \mathrm{~g})$ e EMC (46,7 g), respectivamente. Na fração EMC, observou-se a formação de um precipitado, que foi filtrado a vácuo e submetido a sucessivas lavagens com metanol, fornecendo a substância D-manitol (400 mg). A fração EAC $(5,0 \mathrm{~g})$ foi submetida a uma coluna de gel de sílica, utilizando-se como eluentes hexano, AcOEt e MeOH puros e em misturas binárias, obtendo-se 5 frações, F-H (734 mg), F-HA (2,6 mg), F-A (810 mg), F-AM (512 mg), F-M (208 mg). A fração F-A (810 mg) foi submetida a uma coluna Sephadex LH 20, eluída com metanol, da qual se obtiveram 25 frações de aproximadamente $1 \mathrm{~mL}$. A fração $17-20$ (360 mg) foi cromatografada em gel de sílica, resultando no isolamento do iridoide miopoclorina 3 (30 mg).

\section{Biflorina, 6,9-dimetil-3-(4-metil-3-pentenil)nafto[1,8-bc]-piran- 7,8-diona $(\mathbf{1})$}

Sólido cristalino vermelho escuro; p.f.: $154-157{ }^{\circ} \mathrm{C}$; IV $(\mathrm{KBr}$, $\left.\mathrm{cm}^{-1}\right)$ : 2921; 1684; 1592 e 1438; 1236; 1023; RMN ${ }^{1} \mathrm{H}(500 \mathrm{MHz}$, $\mathrm{CDCl}_{3}$ )- $\delta_{\mathrm{H}}$ (multiplicidade, $J$ em Hz): 1,59 (s, H-14); 1,72 (s, H-15); 1,98 (s, H-17); 2,29 (q, 7,3; H-11); 2,53 (t, 7,3; H-10); 2,70 (s, H-16); 5,16 (s, H-12); 7,07 (s, H-2); 7,39 (d, 8,1; H-5) e 7,52 (d, 8,1; H-4). $\mathrm{RMN}{ }^{13} \mathrm{C}\left(125 \mathrm{MHz}, \mathrm{CDCl}_{3}\right)-\delta_{\mathrm{c}}: 7,8(\mathrm{C}-17) ; 18,0(\mathrm{C}-14) ; 22,6(\mathrm{C}-$ 16); 25,2 (C-15); 26,7 (C-11); 26,7 (C-10); 113,5 (C-9); 116,1 (C-3); 122,0 (C-12); 124, 2 (C-9b); 126,6 (C-6a); 127,8 (C-4); 129,0 (C-3a); 133,6 (C-13); 135,9 (C-5); 140,2 (C-2); 146,7 (C-6); 161,9 (C-9a); 178,0 (C-8); 182,0 (C-7).

\section{Caprariolídeo B, (1R, 5S, 8R) 3-(furan-3'-il)-5,8-dimetil-1-} oxaspiro[4.5]non-2-en-9-ona (2)

Líquido viscoso de cor amarela; $[\alpha]_{\mathrm{D}}^{20}-31^{\circ}\left(c 0,001 ; \mathrm{CHCl}_{3}\right)$; Lit.: $[\alpha]_{\mathrm{D}}^{20}-38^{\circ}$ (c 1,08; $\left.\mathrm{CHCl}_{3}\right) ; \mathrm{IV}\left(\mathrm{KBr}, \mathrm{cm}^{-1}\right): 2922 ; 1760 ; 1454$; 1214; 763; RMN ${ }^{1} \mathrm{H}\left(500 \mathrm{MHz}, \mathrm{CDCl}_{3}\right)-\delta_{\mathrm{H}}$ (multiplicidade, $J$ em Hz): 1,16 (d, 6,0; H-14); 1,32 (d, 7,1; H-15); 1,88 (m, H-6a); 1,89 (m, H-7a); 1,98 (dd, 12,0 e 5,6; H-6b); 2,21 (m, H-5); 2,21 (m, H-4a); 2,56 (dd, 12,5 e 9,2; H-7b); 2,74 (H-4b); 2,94 (m, H-8); 6,11 (s, H-2); 6,45 (s, H-11); 7,40 (s, H-12); 7,43 (s, H-13). RMN ${ }^{13} \mathrm{C}\left(125 \mathrm{MHz}, \mathrm{CDCl}_{3}\right)-\delta_{\mathrm{c}}: 15,7(\mathrm{C}-15) ; 20,5$ (C-14); 29,0 (C-5); 34,3 (C-8); 37,5 (C-4); 42,9 (C-7); 47,5 (C-6); 89,9 (C-1); 110,2 (C-11); 111,9 (C-2); 122,7 (C-10); 141,1 (C-13); 143,5 (C-12); 144,6 (C-3); 179,7 (C-9).

\section{Miopoclorina, (1R,5S,6S,7R,8S,9S)-7-cloro-8-(hidroximetil)octa- hidrociclo-penta[c]piran-1,10-oxira-5,6,8-triol (3) \\ Sólido marrom; $[\alpha]_{\mathrm{D}}^{20}+38^{\circ}\left(\mathrm{c} 0,0006 ;\left(\mathrm{CH}_{3}\right)_{2} \mathrm{CO}\right) ; \mathrm{IV}\left(\mathrm{KBr}, \mathrm{cm}^{-1}\right)$ : $3411 ; 2929 ; 1242 ; 1149 ; 1033$. RMN ${ }^{1} \mathrm{He} \mathrm{e}^{13} \mathrm{C}$ : Tabela 2.}

\section{Reação de acetilação de 3}

A fração 3 (80 mg) foi submetida à reação de acetilação usando metodologia descrita na literatura, ${ }^{12}$ sendo a mesma dissolvida em $1 \mathrm{~mL}$ de piridina e $2 \mathrm{~mL}$ de anidrido acético. Após $24 \mathrm{~h}$ sob agitação à temperatura ambiente, a mistura foi lavada sucessivamente com solução saturada de sulfato de cobre, seca com sulfato de sódio anidro e evaporada sob pressão reduzida. O produto bruto $(61 \mathrm{mg})$ foi submetido à coluna cromatográfica em gel de sílica utilizando $\mathrm{CH}_{2} \mathrm{Cl}_{2}$ como eluente, fornecendo dois produtos acetilados $3 \mathbf{a}(28 \mathrm{mg}-45 \%)$ e $3 \mathbf{b}(19,6 \mathrm{mg}-32 \%)$

\section{Avaliação da atividade larvicida}

Alíquotas das amostras de óleo e hidrolato (10 a $1 \mathrm{mg}$ ) foram dissolvidas em 0,3 mL de dimetilsulfóxido (DMSO) e transferidas para 
um béquer de $50 \mathrm{~mL}$. Posteriormente, foram adicionadas 50 larvas de terceiro estágio, ${ }^{13}$ juntamente com $19,7 \mathrm{~mL}$ de água. Paralelamente, foram feitos testes em branco, utilizando-se água e DMSO a 1,5\%. Após $24 \mathrm{~h}$, foi realizada a contagem das larvas exterminadas e calculada a $\mathrm{CL}_{50}{ }^{14} \mathrm{O} O, O^{\prime}$-(tiodi-4,1-fenileno)bis $(O, O$-dimetilfosforotioato $\left(\right.$ Temephos ${ }^{\circledR}$ ) foi utilizado como padrão positivo do teste.

\section{RESULTADOS E DISCUSSÃO}

As análises por cromatografia gasosa (CG-DIC) e cromatografia gasosa acoplada à espectrometria de massas (CG-EM) possibilitaram a determinação da composição química do óleo essencial (rendimento $0,059 \%$ ) das folhas de C. biflora. A interpretação dos cromatogramas revelou a presença de 16 constituintes químicos, dos quais 14 foram identificados (Tabela 1). Os constituintes apresentaram-se predominantemente representados por terpenoides sesquiterpênicos e o diterpeno fitol. Os constituintes majoritários do óleo de $C$. biflora foram identificados como o trans-cariofileno $(31,1 \%)$ e o $\alpha$-humuleno (43,0\%), correspondendo a $74,1 \%$ da composição total do óleo (Tabela 1). A composição química do óleo essencial de $C$. biflora já foi relatada na literatura, a qual mostrou a identificação de 9 compostos, dentre os quais os majoritários são o $\gamma$-muuroleno $(32,6 \%)$ e o trans-cariofileno $(29,6 \%)$. O $\alpha$-humuleno, que se apresentou como majoritário no presente trabalho, havia representado apenas $6,4 \%$ da composição do óleo no trabalho anterior. $\mathrm{O} \gamma$-muuroleno apresentou apenas 2,24\% da composição deste óleo. ${ }^{15}$ Alguns componentes, como o aromadendreno, germacreno D, 8-isoprenil-1,5-dimetil-ciclodeca-1,5-dieno, $\delta$-cadineno, $E$-nerolidol, germacreno-D-ol, tau-muurolol, $\alpha$-cadinol e fitol, estão sendo reportados pela primeira vez no óleo essencial desta espécie. Tais diferenças em relação à composição química de óleos essenciais de uma mesma espécie são observadas devido a diversos fatores, como local e horário de coleta do material, época do ano, dentre outros.

Tabela 1. Composição química percentual do óleo essencial das folhas de C. biflora

\begin{tabular}{lccc}
\hline Composto $^{\mathrm{a}}$ & $\mathrm{IK}^{\mathrm{b}}$ & $\mathrm{IK}^{\mathrm{c}}$ & Área $(\%)$ \\
\hline$\beta$-elemeno & 1391 & 1391 & 3,57 \\
trans-cariofileno & 1419 & 1419 & 31,11 \\
aromadendreno & 1424 & 1441 & 6,12 \\
$\alpha$-humuleno & 1455 & 1455 & 43,07 \\
$\gamma$-muuroleno & 1485 & 1480 & 2,24 \\
germacreno D & 1495 & 1485 & 1,13 \\
8-isoprenil-1,5-dimetil-ciclodeca-1,5-dieno & 1511 & $\mathrm{ND}$ & 0,31 \\
\&-cadineno & 1528 & 1523 & 0,42 \\
E-nerolidol & 1571 & 1563 & 5,74 \\
germacreno-D-ol & 1585 & 1576 & 0,52 \\
óxido de cariofileno & 1583 & 1583 & 1,56 \\
tau-Muurolol & 1647 & 1642 & 0,28 \\
$\alpha$-cadinol & 1661 & 1654 & 0,29 \\
fitol & 2057 & 1943 & 1,00 \\
\hline Total & & & 97,36 \\
\hline
\end{tabular}

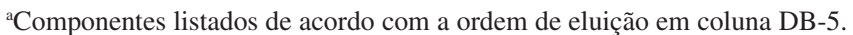

${ }^{\text {b }}$ Índices de Kovats corrigidos. ${ }^{\text { Índices de Kovats da literatura. }{ }^{9} \mathrm{ND}=\text { Não }}$ descrito.

Foram realizados bioensaios com larvas do Aedes aegypti utilizando o óleo essencial e o hidrolato também obtido das folhas de $C$. biflora. O óleo essencial apresentou valor de $\mathrm{CL}_{50}$ igual a $73,39 \mu \mathrm{g} / \mathrm{mL}$ $( \pm 2,27 \mu \mathrm{g} / \mathrm{mL})$, demonstrando uma significante atividade larvicida, uma vez que amostras com valores de $\mathrm{CL}_{50}$ menores que $100 \mu \mathrm{g} / \mathrm{mL}$ são consideradas ativas. ${ }^{16} \mathrm{O}$ hidrolato, entretanto, não apresentou atividade larvicida. Como padrão positivo foi utilizado o composto comercial Temephos $^{\circledast}\left(\mathrm{LC}_{50} 1,4 \pm 0,2 \mu \mathrm{g} / \mathrm{mL}\right)$. A composição química do óleo essencial foi relatada previamente, ${ }^{15}$ entretanto este é o primeiro registro na literatura da atividade larvicida deste óleo. $\mathrm{O}$ óleo essencial foi também submetido ao ensaio antioxidante utilizando DPPH (1,1-difenil-2-picril-hidrazil), entretanto não apresentou nenhuma atividade.

O fracionamento cromatográfico do extrato éter de petróleo das raízes de $C$. biflora permitiu o isolamento de três componentes, que foram identificados como orto-naftoquinona biflorina (1), ${ }^{9}$ caprariolídeo B (2) ${ }^{10}$ e o esteroide $\beta$-sitosterol. ${ }^{2}$ Do extrato etanólico do caule foram isolados e identificados dois constituintes, o carboidrato D-manitol ${ }^{2}$ e o iridoide miopoclorina (3). ${ }^{17} \mathrm{~A}$ acetilação de $\mathbf{3}$ forneceu os derivados acetilados 3a e $\mathbf{3 b}$ (Figura 1).
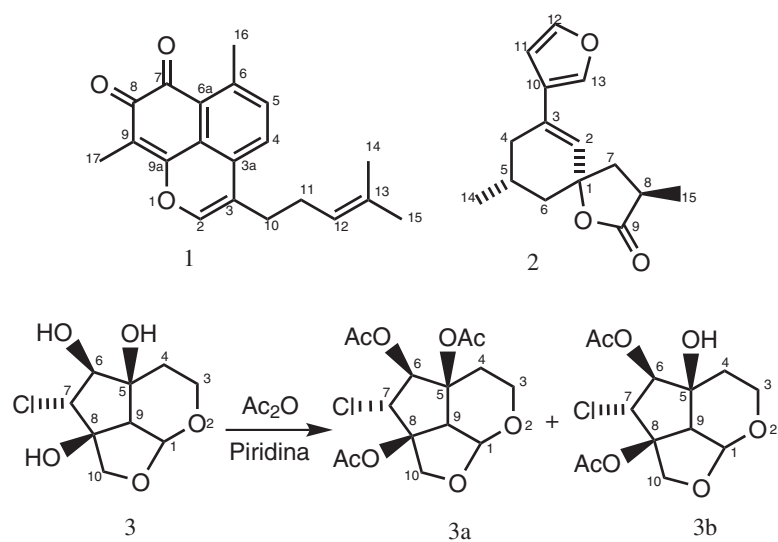

Figura 1. Estrutura dos compostos isolados de Capraria biflora

A quinona biflorina (1), o $\beta$-sitosterol e o D-manitol foram identificados através de comparação dos dados dos espectros de absorção na região do IV e, principalmente, dos dados de $\mathrm{RMN}$ de ${ }^{1} \mathrm{H}$ e ${ }^{13} \mathrm{C}$ com a literatura. ${ }^{2,9}$

O espectro infravermelho (IV) de 2 revelou bandas de absorção em $v_{\text {máx }}\left(\mathrm{cm}^{-1}\right) 1761(\mathrm{C}=\mathrm{O}), 1454(\mathrm{C}=\mathrm{C}), 1214(\mathrm{C}-\mathrm{O})$. A análise dos espectros RMN ${ }^{13} \mathrm{C}-\left\{{ }^{1} \mathrm{H}\right\}$ e RMN ${ }^{13} \mathrm{C}$ - DEPT revelou a presença de quatro sinais de carbonos não hidrogenados [três $\mathrm{sp}^{2}$ e um $\mathrm{sp}^{3}$, sendo um sp ${ }^{3}$ oxigenado em $\delta_{c} 89,95(\mathrm{C}-1)$ e um $\mathrm{sp}^{2}$ carbonílico em $\delta_{c} 179,71(\mathrm{C}-9)$ ]; seis metínicos [quatro $\mathrm{sp}^{2} \mathrm{e}$ dois $\mathrm{sp}^{3}$, sendo dois $\mathrm{sp}^{2}$ oxigenados em $\delta_{\mathrm{c}} 143,54(\mathrm{C}-12)$ e $\delta_{\mathrm{c}} 141,11(\mathrm{C}-13)$ ]; três metilênicos (todos $\mathrm{sp}^{3}$ ) e dois metílicos. $\mathrm{O}$ espectro de $\mathrm{RMN}{ }^{1} \mathrm{H}$ mostrou quatro sinais de hidrogênios ligados a carbonos olefínicos, sendo dois carbonos oxigenados $\left[\delta_{\mathrm{H}} 7,40(\mathrm{~s}, \mathrm{H}-12)\right.$ e $\left.\delta_{\mathrm{H}} 7,43(\mathrm{~s}, \mathrm{H}-13)\right]$. Com base nestes dados foi possível propor para o composto 2 a estrutura de um sesquiterpeno, já reportado nesta espécie, conhecido como caprariolídeo B. A comparação dos dados espectrais com valores da literatura confirmou a estrutura do caprariolídeo B. ${ }^{10}$

$\mathrm{O}$ espectro IV de 3 revelou bandas em $v_{\text {máx }}\left(\mathrm{cm}^{-1}\right) 3411(\mathrm{OH})$, 2929 (C-H), 1149 e 1033 (C-O).

Os espectros de $\mathrm{RMN}{ }^{1} \mathrm{H}$ (1D e $2 \mathrm{D}{ }^{1} \mathrm{H}-{ }^{1} \mathrm{H}-\mathrm{COSY}$ ) apresentaram sinais correspondentes a um hidrogênio metínico ligado a carbono carbinólico $\left[\delta_{\mathrm{H}} 3,76(1 \mathrm{H}, \mathrm{d}, J=11,0 ; \mathrm{H}-6)\right]$ interagindo com um vizinho em $\delta_{\mathrm{H}} 4.55(1 \mathrm{H}, \mathrm{d}, J=11,0 ; \mathrm{H}-7)$, dois metilênicos heterotópicos ligados a carbonos oxigenados, sendo um isolado em $\delta_{\mathrm{H}}$ $4,39(1 \mathrm{H}, \mathrm{d}, J=9,0 ; \mathrm{H}-1)$ e $3,40(1 \mathrm{H}, \mathrm{dd}, J=9,0 ; 1,5 ; \mathrm{H}-10 \mathrm{~b})$ e um em $\delta_{\mathrm{H}} 3,70(1 \mathrm{H}, \mathrm{dt}, J=13,7 ; 3,7 ; \mathrm{H}-3 \mathrm{a})$, e $3,61(1 \mathrm{H}, \mathrm{ddd}, J=13,7$; 5,$0 ; 3,7 ; \mathrm{H}-3 \mathrm{~b}$ ) envolvendo acoplamento com os $2 \mathrm{H}-4$, e um ligado a 
Tabela 2. Correlação heteronuclear ${ }^{1} \mathrm{H},{ }^{13} \mathrm{C}-\mathrm{HSQC}\left(500 \mathrm{X} 125 \mathrm{MHz}, \mathrm{CDCl}_{3}\right)$ de $\mathbf{3}$, 3a e $\mathbf{3 b}$

\begin{tabular}{|c|c|c|c|c|c|c|}
\hline \multicolumn{7}{|c|}{ HSQC } \\
\hline \multirow{2}{*}{$\mathrm{C}$} & \multicolumn{2}{|l|}{3} & \multicolumn{2}{|l|}{$3 a$} & \multicolumn{2}{|l|}{$3 b$} \\
\hline & $\delta_{\mathrm{H}}$ & $\delta_{\mathrm{C}}$ & $\delta_{\mathrm{H}}$ & $\delta_{\mathrm{C}}$ & $\delta_{\mathrm{H}}$ & $\delta_{\mathrm{C}}$ \\
\hline 1 & $5,39(\mathrm{~d} ; 5,0)$ & 101,7 & $5,63(\mathrm{~d} ; 6,0)$ & 100,9 & $5,68(\mathrm{~d} ; 5,6)$ & 100,5 \\
\hline 3 & $\begin{array}{c}3,70(\mathrm{dt} ; 13,7 ; 3,7) \\
3,61(\mathrm{ddd} ; 13,7 ; 5,0 ; 3,7)\end{array}$ & 57,9 & $\begin{array}{c}4,01(\mathrm{dt} ; 12,5 ; 2,0) \\
3,75(\mathrm{~m})\end{array}$ & 57,6 & $\begin{array}{c}4,03(\mathrm{dt} ; 12,5 ; 2,5) \\
3,82(\mathrm{ddd} ; 12,5 ; 5,2 ; 2,0)\end{array}$ & 57,7 \\
\hline 4 & $\begin{array}{c}1,84(\mathrm{dl} ; 15,0) \\
1,58(\mathrm{dt} ; 15,0 ; 5,0)\end{array}$ & 31,9 & $\begin{array}{c}2,46(\mathrm{~m}) \\
1,86(\mathrm{~d} ; 14,0)\end{array}$ & 26,8 & $\begin{array}{c}1,81(\mathrm{~m}) \\
1,72(\mathrm{dd} ; 11,7 ; 2,0)\end{array}$ & 30,9 \\
\hline 5 & - & 72,8 & - & 81,6 & - & 75,0 \\
\hline 6 & $3,76(\mathrm{~d} ; 11,0)$ & 76,5 & $5,35(\mathrm{~d} ; 11,5)$ & 74,0 & $5,35(\mathrm{~d} ; 11,2)$ & 73,2 \\
\hline 7 & $4,55(\mathrm{dd} ; 11,0 ; 1,5)$ & 73,0 & $5,11(\mathrm{dd} ; 11,5 ; 1,0)$ & 63,5 & $5,12(\mathrm{dd} ; 11,2 ; 1,5)$ & 64,5 \\
\hline 8 & - & 84,0 & - & 88,1 & - & 88,5 \\
\hline 9 & $2,19(\mathrm{~d} ; 5,0)$ & 55,2 & $3,49(\mathrm{~d} ; 6,0)$ & 47,4 & $2,78(\mathrm{~d} ; 5,6)$ & 51,4 \\
\hline 10 & $\begin{array}{c}4,39(\mathrm{~d} ; 9,0) \\
3,40(\mathrm{dd} ; 9,0 ; 1,5) \mathrm{W} \operatorname{com} 7\end{array}$ & 71,8 & $\begin{array}{c}4,61(\mathrm{~d} ; 10,5) \\
3,73(\mathrm{dd} ; 10,5 ; 1,5)\end{array}$ & 68,7 & $\begin{array}{c}4,72(\mathrm{~d} ; 10,5) \\
3,80(\mathrm{dd} ; 10,5 ; 1,5)\end{array}$ & 68,0 \\
\hline $11-\mathrm{Ac}$ & - & - & - & 169,7 & - & - \\
\hline $12-\mathrm{Ac}$ & - & & $2,06(\mathrm{~s})$ & 21,7 & - & - \\
\hline $13-\mathrm{Ac}$ & - & - & - & 170,1 & - & 170,3 \\
\hline $14-\mathrm{Ac}$ & - & - & $2,10(\mathrm{~s})$ & 22,5 & $2,18(\mathrm{~s})$ & 21,9 \\
\hline 15-Ac & - & - & - & 170,4 & - & 171,1 \\
\hline $16-\mathrm{Ac}$ & - & - & $2,15(\mathrm{~s})$ & 20,6 & $2,25(\mathrm{~s})$ & 20,8 \\
\hline
\end{tabular}

*O número de átomos de hidrogênio ligados a carbono foi deduzido com base na análise comparativa dos espectros de RMN ${ }^{13} \mathrm{C}\left\{{ }^{1} \mathrm{H}\right\}$ e RMN ${ }^{13} \mathrm{C}-\mathrm{DEPT}$. Espectro $2 \mathrm{D}{ }^{1} \mathrm{H}-{ }^{1} \mathrm{H}$-COSY foi também usado na atribuição dos deslocamentos químicos. Os valores dos deslocamentos químicos e das constantes de acoplamento $(J)$ dos átomos de hidrogênio foram obtidos do espectro 1D de RMN ${ }^{1} \mathrm{H}$. Espectros $2 \mathrm{D}$ HMBC foram também usados na interpretação destes dados espectrais.

carbono acetálico em $\delta_{\mathrm{H}} 5,39(1 \mathrm{H}, \mathrm{d}, J=5,0)$. Estes sinais revelaram-se compatíveis com a presença de esqueleto de iridoide (Tabela 2).

$\mathrm{O}$ espectro de $\mathrm{RMN}^{13} \mathrm{C}$ de 3 permitiu identificar a presença de sinais referentes a nove átomos de carbono, sendo um deles o carbono acetálico $\left(\delta_{\mathrm{C}} 101,7, \mathrm{CH}-1\right)$ presente na estrutura de um iridoide. $\mathrm{O}$ espectro de massas (EM-IES), registrado no modo negativo, revelou picos do íon molecular [M+Cl]', em $\mathrm{m} / \mathrm{z}, 271,0231, \mathrm{~m} / \mathrm{z}, 273,0110$ (calculado) e $\mathrm{m} / z$ 275,0081 (calculado), previstos numa relação de intensidade relativa de 9:6:1 com base na abundância natural 3:1 de ${ }^{35} \mathrm{Cl} /{ }^{37} \mathrm{Cl}$, correspondendo aos picos $[\mathrm{M}]$, [M+2] e [M+4]. Estes dados sugeriram a presença de um átomo de cloro na molécula original, compatível com os $\delta_{\mathrm{C}} 73,0$ e $\delta_{\mathrm{H}} 4,55$ do $\mathrm{CH}-7$ (Tabela 2) correlacionados pela correspondente interação heteronuclear deduzida pelo espectro 2D HSQC. A localização do cloro foi confirmada através de uma reação de acetilação, verificando-se a formação dos dois produtos acetilados 3a e 3b (Figura 1), um triacetilado nos carbonos 5, 6 e 8 (3a) e um diacetilado nos carbonos 6 e 8 (3b). Dados espectroscópicos adicionais ( $\mathrm{RMN}{ }^{1} \mathrm{H},{ }^{13} \mathrm{C}$ e EM-IES) corroboraram a proposta estrutural e possibilitaram a localização do cloro no carbono C-7. Os fragmentos que justificam os principais picos observados no espectro de massas de $\mathbf{3}$ encontram-se ilustrados na Figura 2.

Os derivados acetilados $\mathbf{3} \mathbf{a}$ e $\mathbf{3 b}$ confirmaram a proposta estrutural de $\mathbf{3}$, e foram usados também para a determinação da configuração relativa deste composto.

A configuração relativa inferida para os grupos hidroxilas e acetilas presentes nas moléculas de $\mathbf{3}, \mathbf{3 a}$ e $\mathbf{3 b}$ foram deduzidas através do espectro NOE por diferença de espectro de RMN ${ }^{1} \mathrm{H}$, obtido a partir de $3 \mathbf{b}$, o qual revelou interações espaciais entre os hidrogênios H-6 $\left(\delta_{\mathrm{H}} 5,35\right), \mathrm{H}-10 \mathrm{a}\left(\delta_{\mathrm{H}} 4,72\right)$ е H-3a $\left(\delta_{\mathrm{H}} 4,03\right)$, о H-7 $\left(\delta_{\mathrm{H}} 5,12\right)$ е о H-9 $\left(\delta_{\mathrm{H}} 2,78\right)$ e H-1 $\left(\delta_{\mathrm{H}} 5,62\right)$ com os hidrogênios H-3b $\left(\delta_{\mathrm{H}} 3,82\right)$ e $\mathrm{H}-9\left(\delta_{\mathrm{H}} 2,78\right)$ (Figura 3$)$. Estas interações espaciais sugeriram uma configuração $\beta$ para a hidroxila no carbono C-5 e o grupo acetila no carbono C-8. Assim, a configuração relativa de cada centro estereogênico foi determinada com base nas interações, entre dois núcleos de hidrogênio (dipolar-dipolar), observadas no espectro NOE, entre o

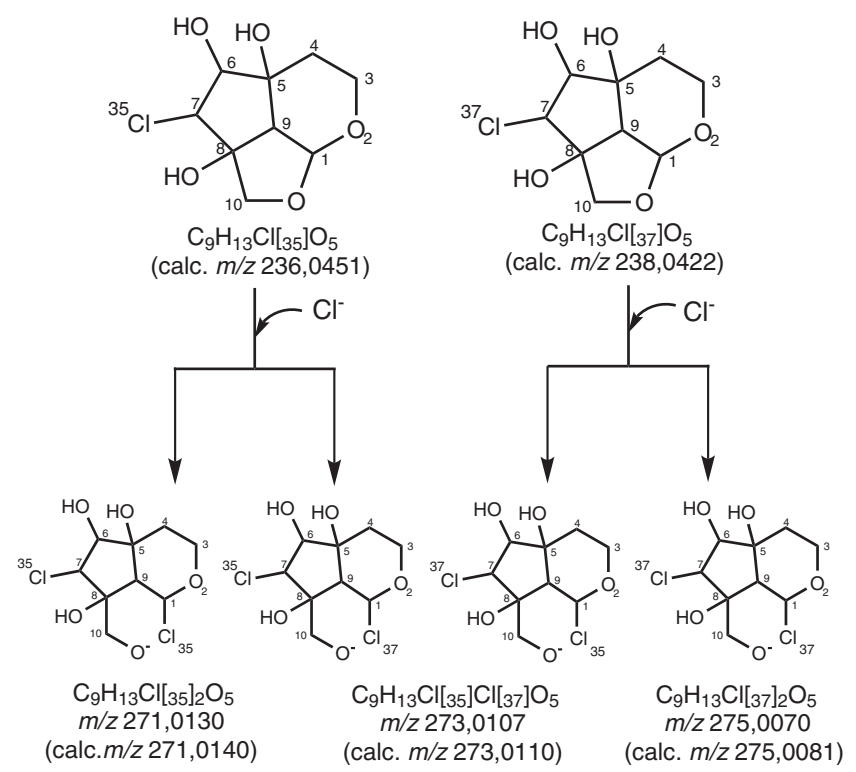

Figura 2. Proposta mecanística para os principais picos observados no espectro de massas de 3

hidrogênio H-1 $\left(\delta_{\mathrm{H}} 5,62\right)$ com os hidrogênios H-3b $\left(\delta_{\mathrm{H}} 3,82\right)$, H-10b $\left(\delta_{\mathrm{H}} 3,80\right)$ e H-9 $\left(\delta_{\mathrm{H}} 2,78\right)$, do hidrogênio H-6 $\left(\delta_{\mathrm{H}} 5,35\right)$ com os hidrogênios H-10a $\left(\delta_{\mathrm{H}} 4,72\right)$ e H-3a $\left(\delta_{\mathrm{H}} 4,03\right)$ e do hidrogênio H-7 $\left(\delta_{\mathrm{H}}\right.$ $5,12)$ com o hidrogênio H-9 $\left(\delta_{\mathrm{H}} 2,78\right)$ (Figura 3 ).

A reação de acetilação e comparação com dados da litera$\operatorname{tura}^{17,18}$ permitiram a confirmação da proposta estrutural para a referida substância com sua respectiva estereoquímica, que foi identificada como a $(1 R, 5 S, 6 S, 7 R, 8 S, 9 S)$-7-cloro-8-(hidroximetil) octa-hidrociclopenta[c]piran-1,10-oxira-5,6,8-triol (miopoclorina), já identificada anteriormente na espécie Myoporum bontioides e que está sendo reportada pela primeira vez em $C$. biflora, bem como seus derivados acetilados. 


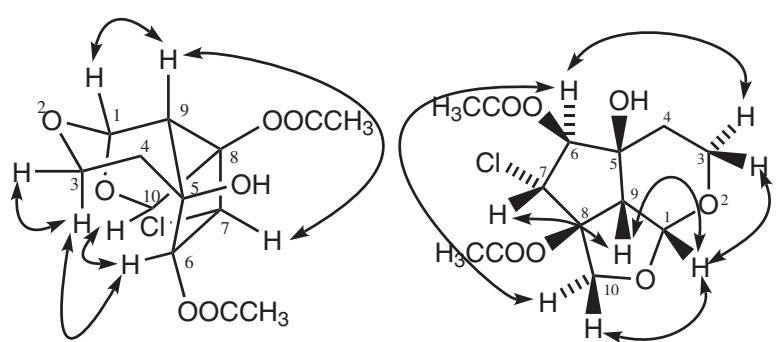

Figura 3. Interações espaciais entre os hidrogênios do derivado diacetilado determinando a configuração relativa da miopoclorina

\section{MATERIAL SUPLEMENTAR}

Os espectros IV, EM, RMN ${ }^{13} \mathrm{C}, \mathrm{RMN}{ }^{1} \mathrm{H}, \mathrm{NOE}, \mathrm{HSQC}$ e HMBC da substância isolada miopoclorina e seus derivados acetilados, bem como os cromatogramas e espectros de massas do óleo essencial, encontram-se disponíveis em http://quimicanova.sbq.org.br, em arquivo pdf, com acesso livre.

\section{AGRADECIMENTOS}

Aos órgãos de fomento à pesquisa CNPq, CAPES, FUNCAP pelas bolsas e auxílios concedidos, ao CENAUREMN-UFC pelos espectros de RMN, ao LEMANOR-UFC pelos espectros de massa de alta resolução, ao Programa de Pós-Graduação em Química (UFC) e FAPERJ pela Bolsa de Pesquisador Visitante Emérito concedida a R. Braz-Filho.

\section{REFERÊNCIAS}

1. Mabberley, D. J.; The plant-book: a portable dictionary of the vascular plants, $2^{\text {nd }}$ ed., Cambridge University Press: Cambridge, 1997.

2. Fonseca, A. M.; Dissertação de Mestrado, Universidade Federal do Ceará, Brasil, 2003.

3. Aquino, T. M.; Lima, C. S. A.; Albuquerque, U. P.; Amorim, E. L. C.; Acta Farm. Bonaerense 2006, 25, 460.

4. Matos, F. J. A.; Plantas Medicinais, Universidade Aberta: Fortaleza, 1988.

5. Aquino, T. M.; Dissertação de Mestrado, Universidade Federal de Pernambuco, Brasil, 2003.
6. Lima, O. G.; D'albuquerque, I. L.; Loureiro, P.; Carmona, C. L.; Bernard, M. C.; Rev. Quím. Ind. 1953, 22, 249.

7. Lima, O. G.; D'albuquerque, I. L.; Loureiro, P.; An. Soc. Biol. Pe. 1953, $X I, 3$.

8. Vasconcellos, M. C.; Bezerra, D. P.; Fonseca, A. M.; Araujo, A. J.; Pessoa, C.; Lemos, T. L. G.; Costa-Lotufo, L. V.; Moraes, M. O.; Montenegro, R. C.; Melanoma Research 2011, 21, 106; Vasconcellos, M. C.; Moura, D. J.; Rosa, R. M.; Machado, M. S.; Guecheva, T. N.; Villela, I.; Immich, B. F.; Montenegro, R. C.; Fonseca, A. M.; Lemos, T. L. G.; Arch. Toxicol. 2010, 84, 799; Vasconcelos, M. C.; Bezerra, D. P.; Fonseca, A. M.; Pereira, M. R. P.; Lemos, T. L. G.; Pessoa, O. D. L.; Pessoa, C.; Moraes, M. O.; Alves, A. P. N. N.; Costa-Lotufo, L. V.; Biol. Pharm. Bull. 2007, 30, 1416; Acosta, S. L.; Muro, L. V.; Sacerio, A. L.; Pena, A. R.; Okwei, S. N.; Fitoterapia 2003, 74, 686; Lima, O. G.; Albuquerque, I. L.; Bento, M. N.; Albuquerque, M. M.; Rev. Inst. Antibióticos 1958, 1, 95.

9. Fonseca, A. M.; Silveira, E. R.; Pessoa, O. D. L.; Lemos, T. L. G.; Magn. Reson. Chem. 2002, 41, 1038.

10. Collins, D. O.; Gallimore, W. A.; Reynolds, W. F.; Williams, L. A. D.; Reese, P. B.; J. Nat. Prod. 2000, 63, 1515.

11. Adams, R. P.; Identification of Essential Oils Components by Gas Chromatography/ Mass Spectrometry, Allured Publ. Corp.: Carol Steam, 2007.

12. Almeida, M. C. S.; Alves, L. A.; Souza, L. G. S.; Machado, L. L.; de Mattos, M. C.; de Oliveira, M. C. F.; Lemos, T. L. G.; Braz-Filho, R.; Quim. Nova 2010, 33, 1877.

13. Gadelha, D. P.; Toda, A. T.; Rev. Bras. Malariol. D. Trop. 1985, 37, 29.

14. Oliveira, M. F.; Lemos, T. L. G.; de Mattos, M. C.; Segundo, T. A.; Santiago, G. M. P.; Braz-Filho, R.; An. Acad. Bras. Cienc. 2002, 74, 211.

15. Fonseca, A. M.; Pessoa, O. D. L.; Lemos, T. L. G.; Nascimento, R. F.; J. Essent. Oil Res. 2006, 18, 157.

16. Cheng, S. S.; Chang, H. T.; Chang, S. T.; Tsai, K. H.; Chen, W. J.; Biosensor. Technol. 2003, 89, 99.

17. Kanemoto, M.; Matsunamia, K.; Otsuka, H.; Shinzato, T.; Ishigaki, C.; Takeda, Y.; Phytochemistry 2008, 69, 2517.

18. Wang, H.; Wu, F. H.; Xiong, F.; Wu, J. J.; Zhang, L. Y.; Ye, W. C.; Li, P.; Zhao, S. X.; Chem. Pharm. Bull. 2006, 54, 1144. 


\section{CONSTITUINTES QUÍMICOS DE Capraria biflora (Scrophulariaceae) E ATIVIDADE LARVICIDA DE SEU ÓLEO} ESSENCIAL ${ }^{\#}$

Luciana Gregório da S. Souza, Macia Cleane S. Almeida, Francisco José Q. Monte, Gilvandete Maria P. Santiago, Raimundo Braz-Filho"\# e Telma Leda G. Lemos*

Departamento de Química Orgânica e Inorgânica, Universidade Federal do Ceará, Campus do Pici, 60451-970 Fortaleza - CE, Brasil Clerton L. Gomes e Ronaldo F. do Nascimento

Departamento de Química Analítica e Físico-Química, Centro de Cências, Universidade Federal do Ceará, Campus do Pici, 60451-970 Fortaleza - CE, Brasil

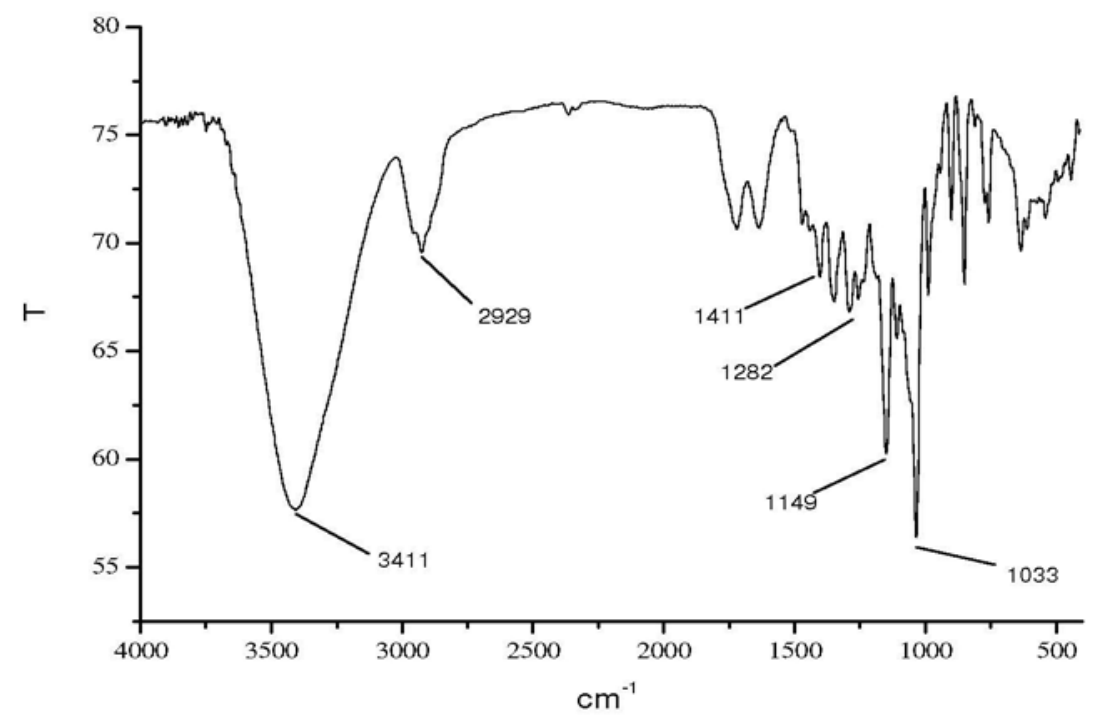

Figura 1S. Espectro na região do infravermelho $(\mathrm{KBr})$ de $\mathbf{3}$ (miopoclorina)
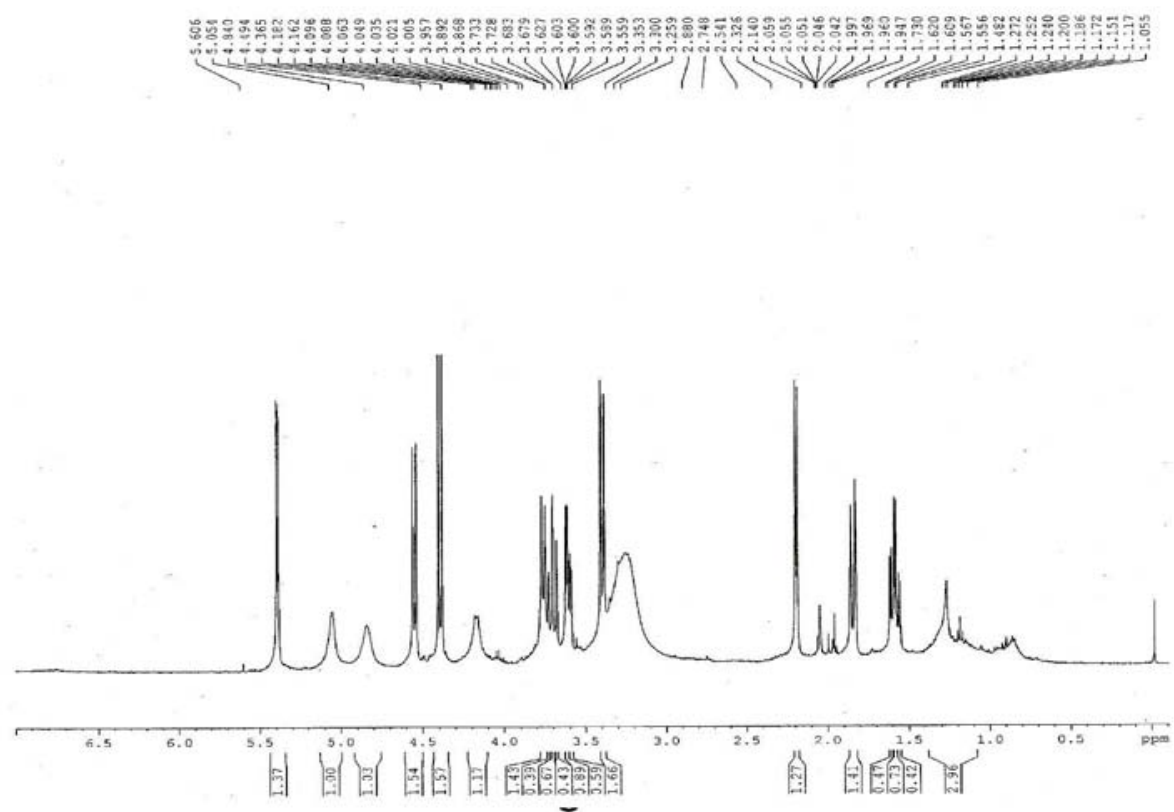

Figura $2 \mathrm{~S}$. Espectro de $\mathrm{RMN}{ }^{1} \mathrm{H}\left[500 \mathrm{MHz},\left(\mathrm{CD}_{3}\right)_{2} \mathrm{CO}\right]$ de 3 (miopoclorina)

*e-mail: tlemos@dqoi.ufc.br

\#Artigo em homenagem ao Prof. Otto R. Gottlieb (31/8/1920-19/6/2011)

\# Pesquisador Visitante Emérito - FAPERJ/UENF/UFRRJ 

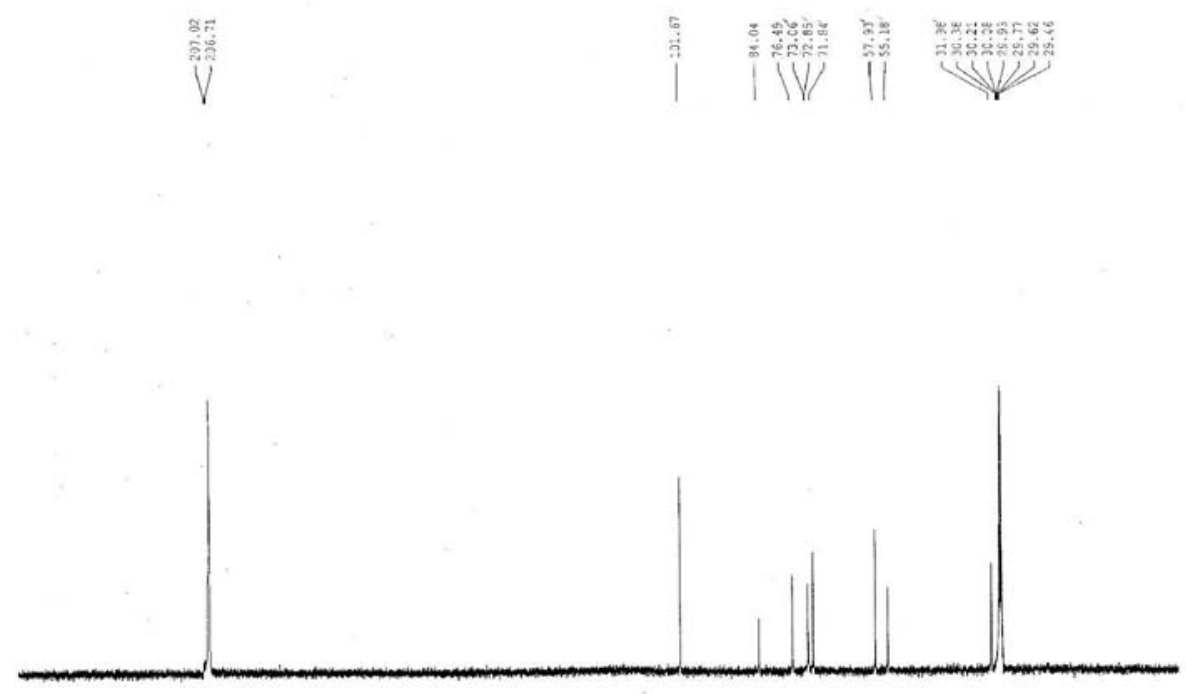

Figura 3S. Espectro de $\mathrm{RMN}^{13} \mathrm{C}\left[125 \mathrm{MHz},\left(\mathrm{CD}_{3}\right)_{2} \mathrm{CO}\right]$ de $\mathbf{3}$ (miopoclorina)

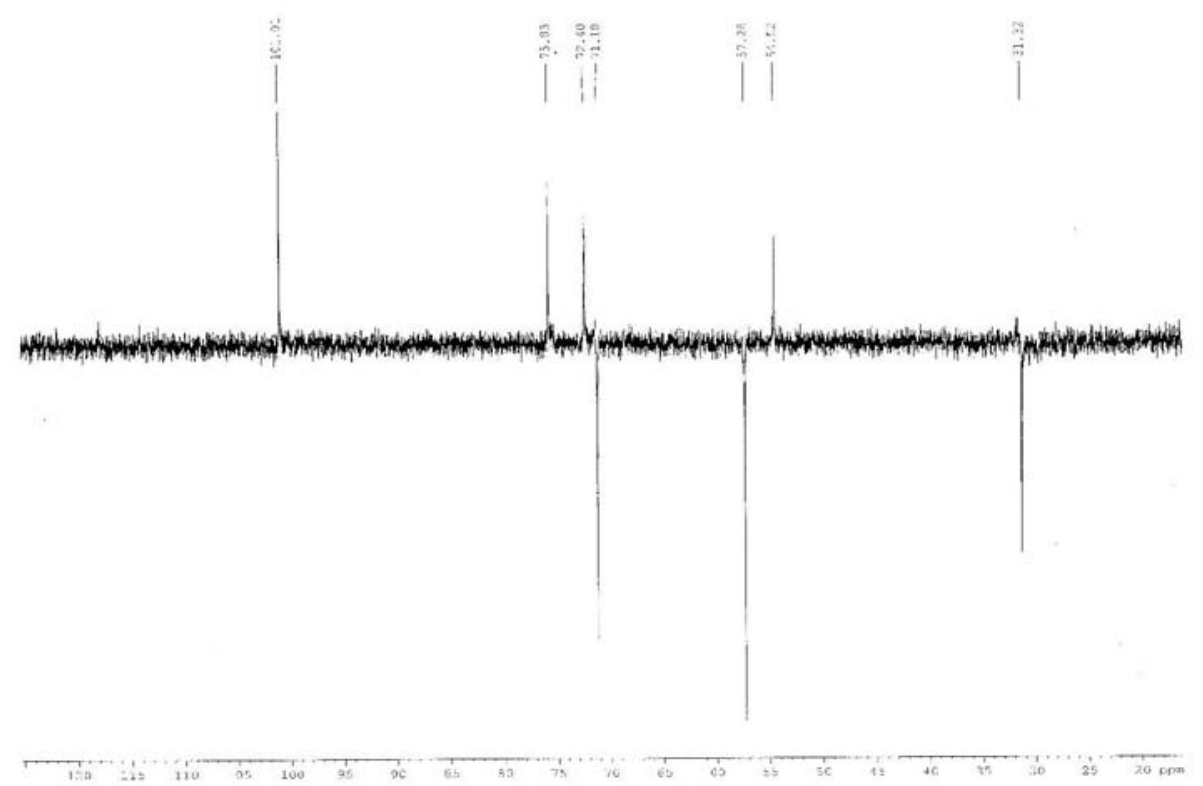

Figura 4S. Espectro de $R M N^{13} \mathrm{C}$-DEPT $135^{\circ}\left[125 \mathrm{MHz},\left(\mathrm{CD}_{3}\right)_{2} \mathrm{CO}\right]$ de 3 (miopoclorina) 


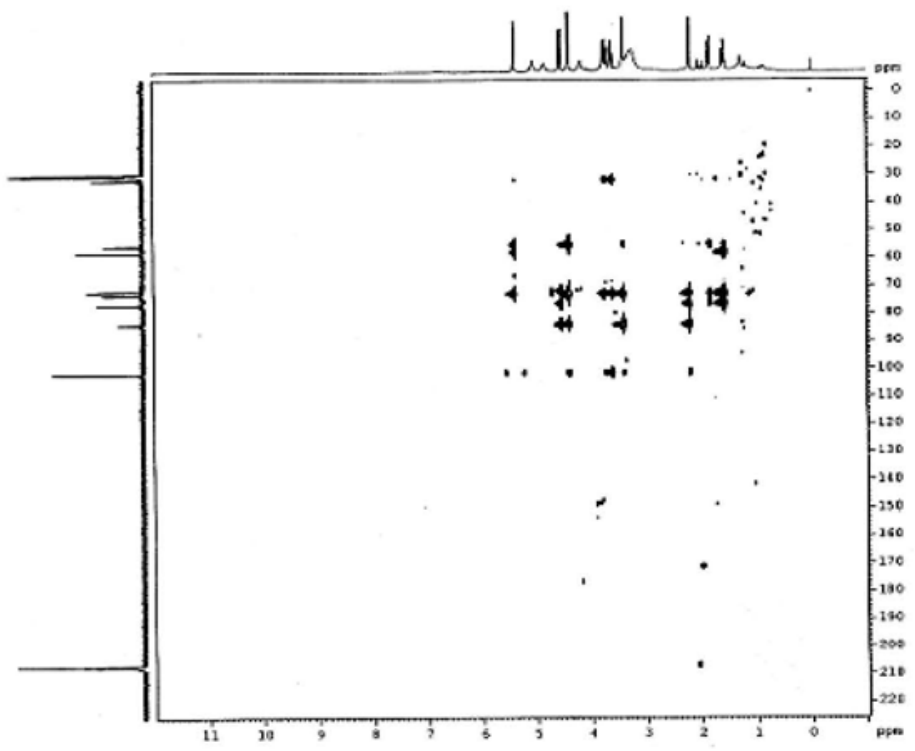

Figura 5S. Espectro de $\mathrm{RMN}{ }^{1} \mathrm{H},{ }^{13} \mathrm{C}-\mathrm{HMBC}\left[500 \mathrm{MHz},\left(\mathrm{CD}_{3}\right)_{2} \mathrm{CO}\right]$ de 3 (miopoclorina)

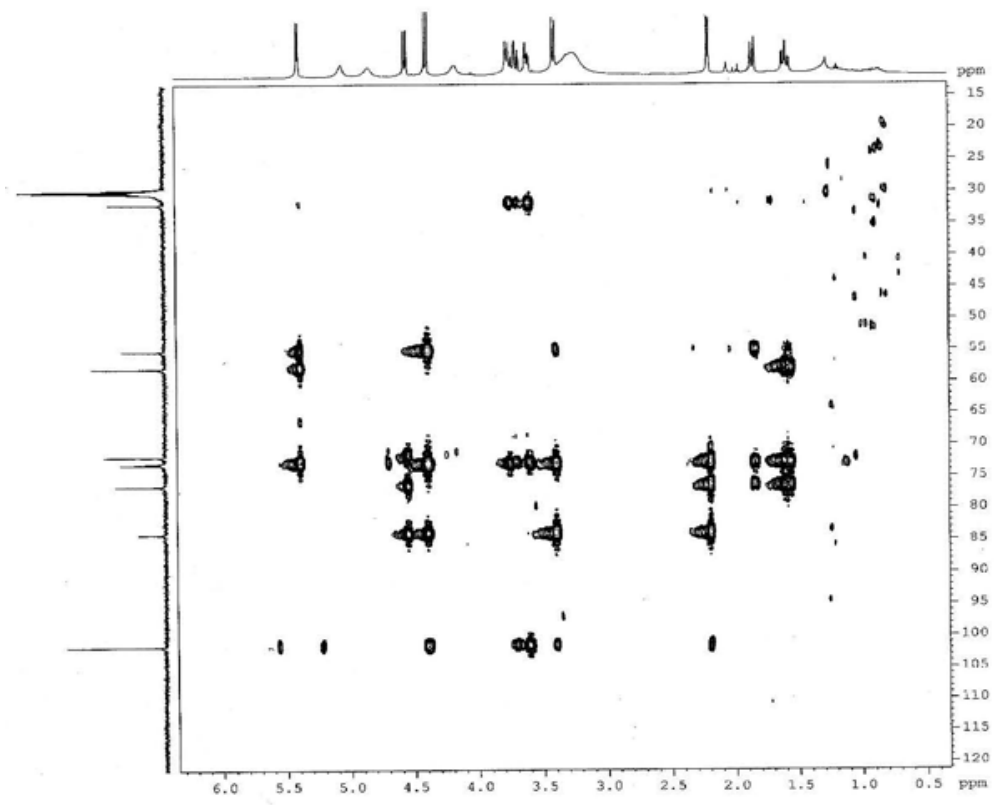

Figura 6S. Expansão (0,5 a 6 ppm para ${ }^{1} \mathrm{H}$ e 15 a 120 ppm para $\left.{ }^{13} \mathrm{C}\right)$ do espectro de $\mathrm{RMN}{ }^{1} \mathrm{H},{ }^{13} \mathrm{C}-\mathrm{HMBC}\left[500 \mathrm{MHz},(\mathrm{CD})_{2} \mathrm{CO}\right]$ de 3 (miopoclorina)

MSMS: Precursor m/z ..... /. Base Peak 349.03(10797333)

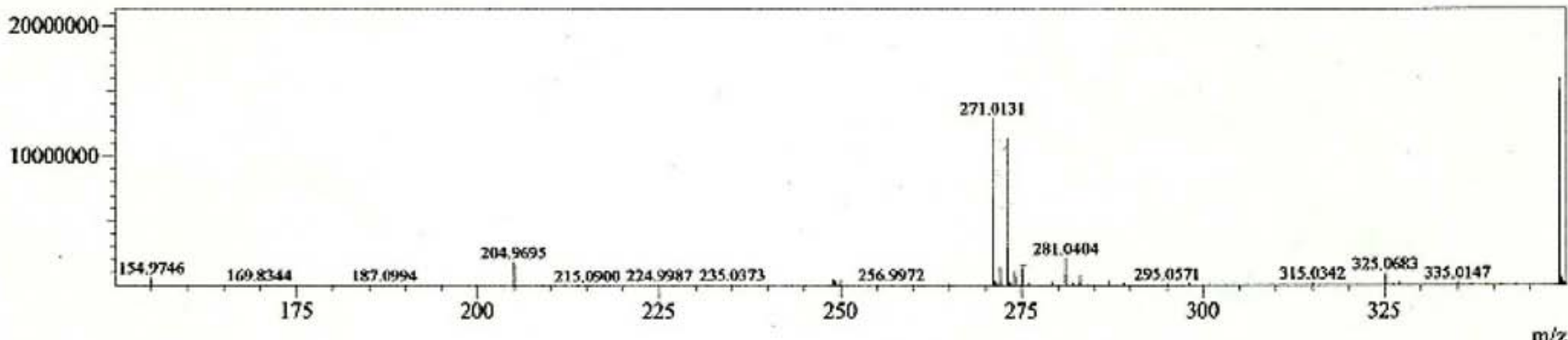

Figura 7S. Espectro de massa de alta resolução EM-IES (modo negativo) de 3 (miopoclorina) 


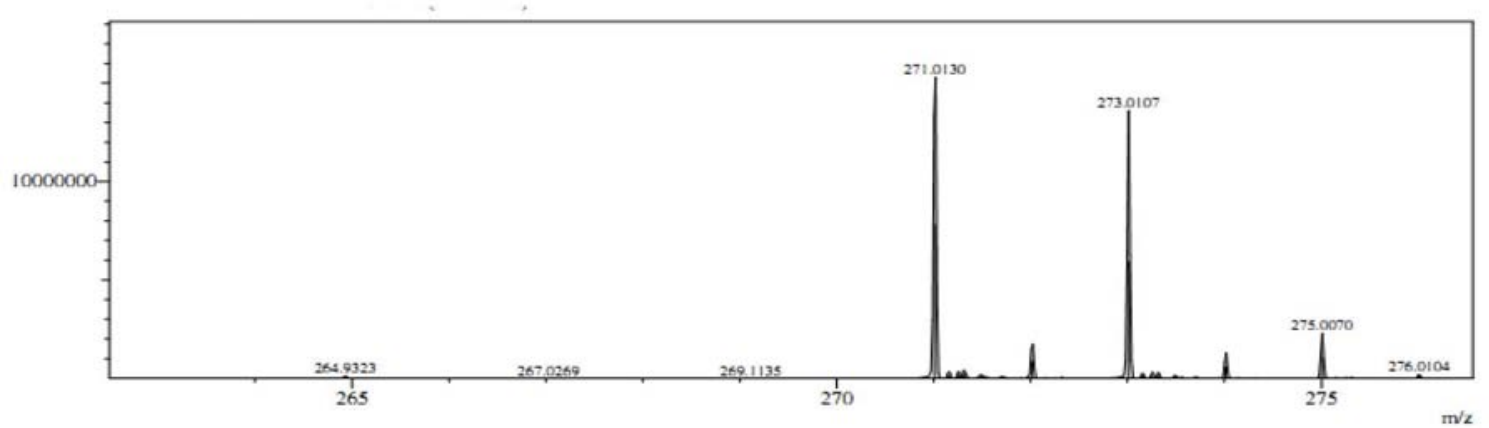

Figura 8S Expansão do espectro de massa de alta resolução EM-IES (modo negativo) de 3 (miopoclorina)

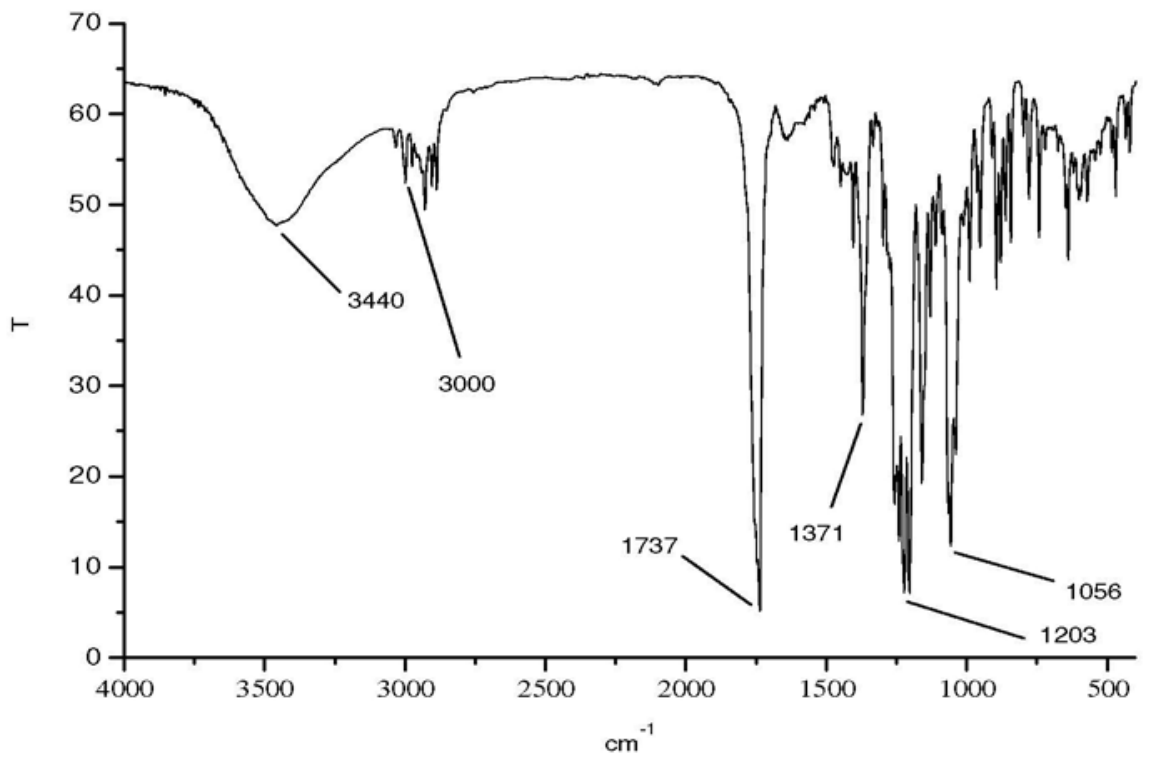

Figura $9 \mathrm{~S}$ Espectro na região do infravermelho do derivado triacetilado da miopoclorina ( $\mathrm{KBr}$ )

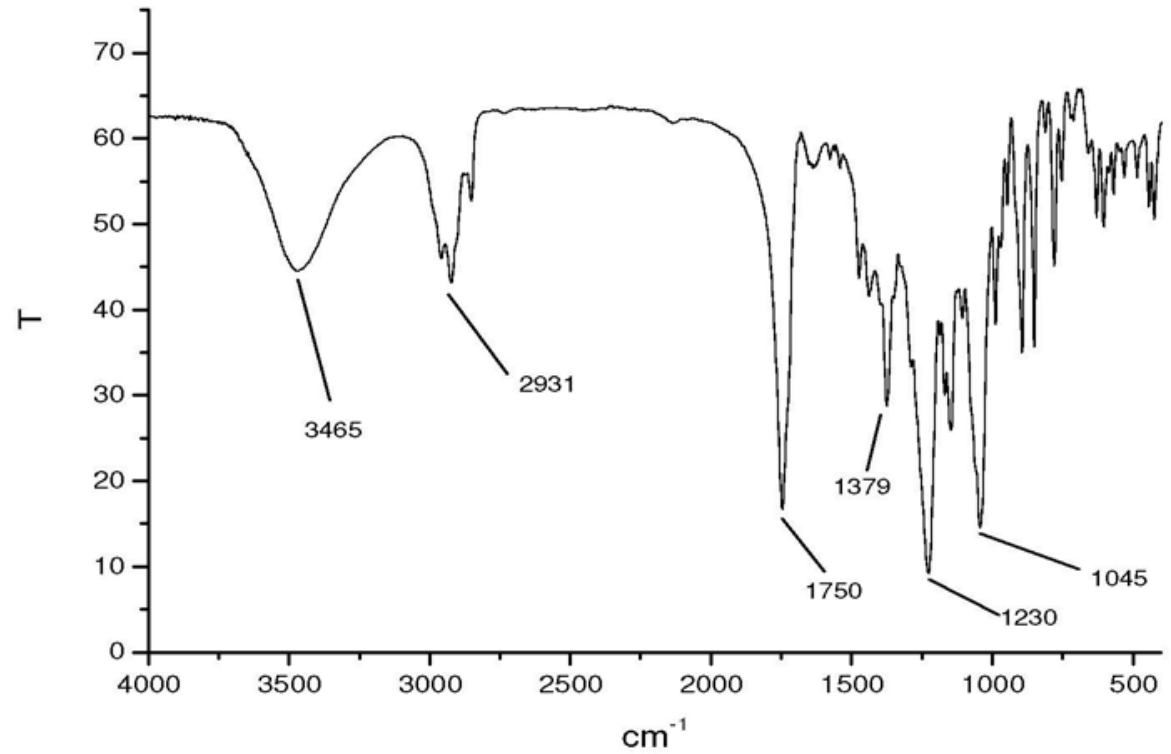

Figura $10 S$ Espectro na região do infravermelho do derivado diacetilado da miopoclorina ( $\mathrm{KBr}$ ) 


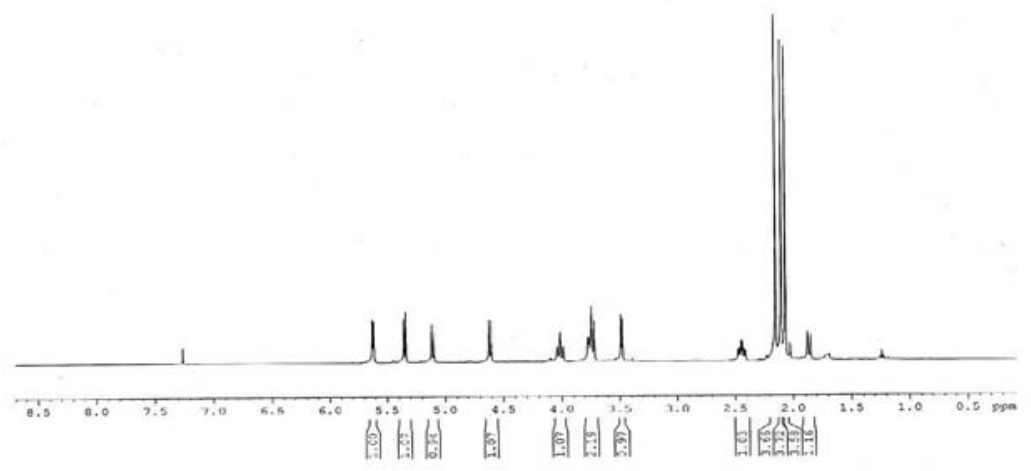

Figura 11S. Espectro de $\mathrm{RMN}^{1} \mathrm{H}\left[500 \mathrm{MHz}, \mathrm{CDCl}_{3}\right]$ do derivado triacetilado da miopoclorina

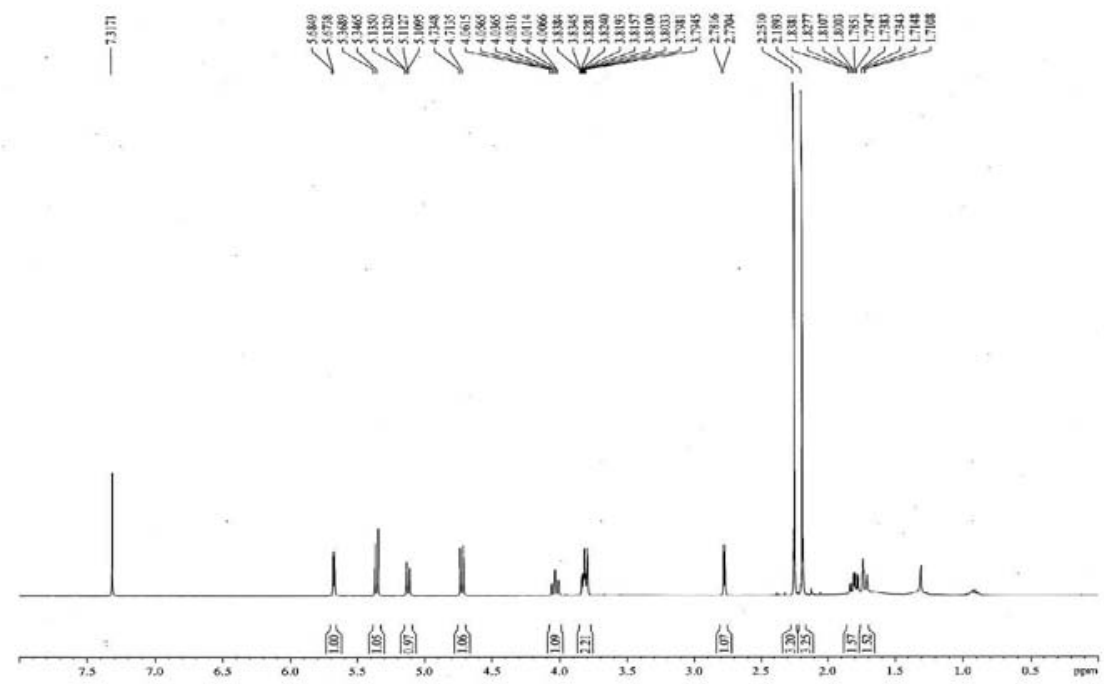

Figura 12S. Espectro de $\mathrm{RMN}^{1} \mathrm{H}\left[500 \mathrm{MHz}, \mathrm{CDCl}_{3}\right]$ do derivado diacetilado da miopoclorina
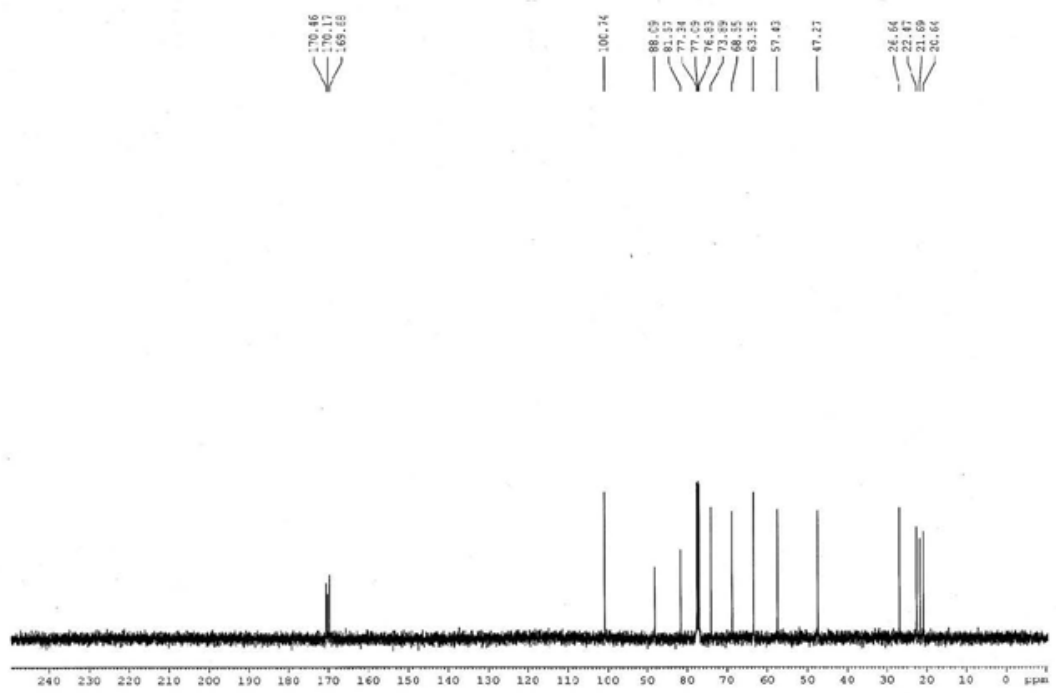

Figura 13S. Espectro de $\mathrm{RMN}^{13} \mathrm{C}-\mathrm{BB}\left[125 \mathrm{MHz}, \mathrm{CDCl}_{3}\right]$ do derivado triacetilado da miopoclorina 


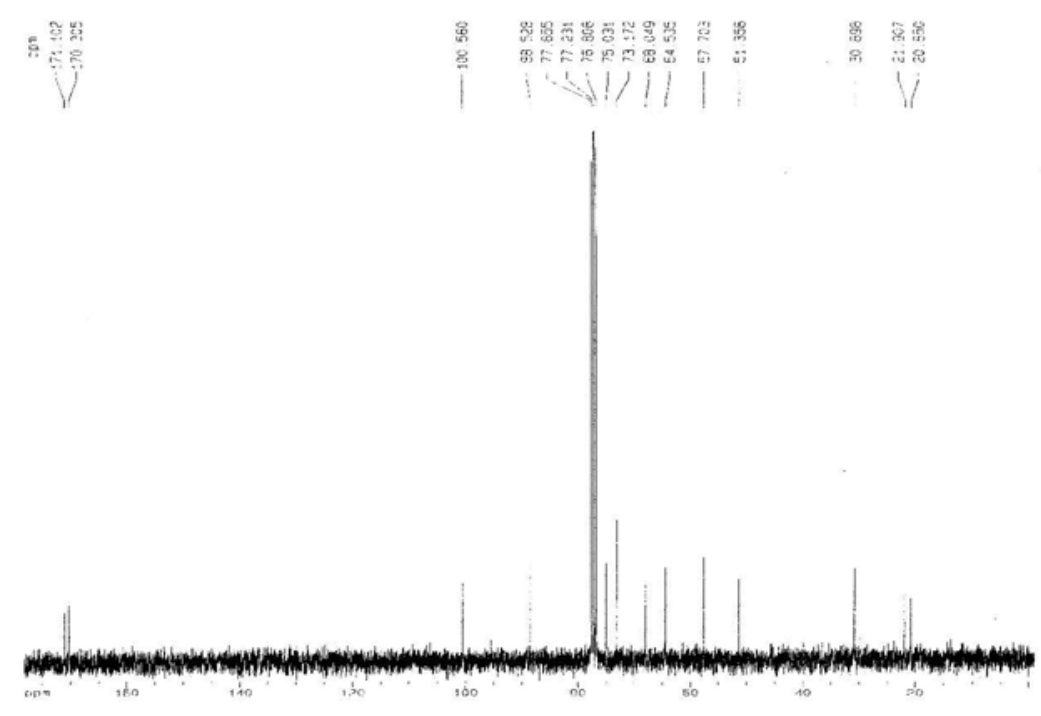

Figura 14S. Espectro de $\mathrm{RMN}{ }^{13} \mathrm{C}-\mathrm{BB}\left[125 \mathrm{MHz}, \mathrm{CDCl}_{3}\right]$ do derivado diacetilado da miopoclorina

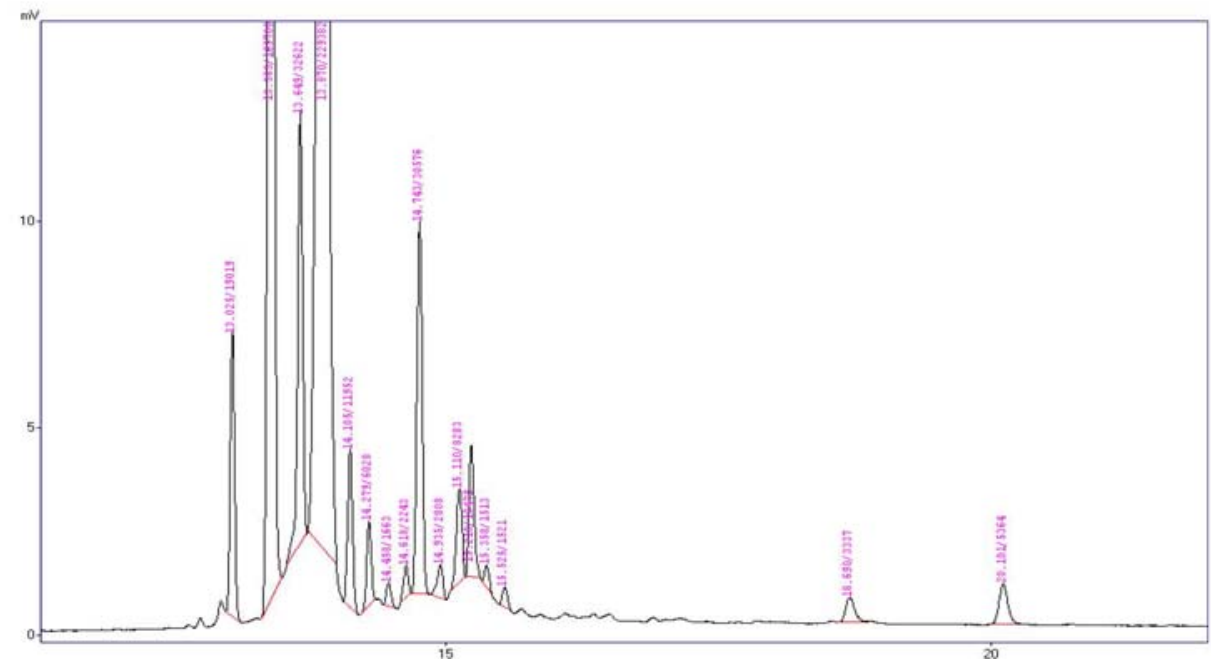

Figura 15S. Cromatograma CG-DIC do óleo essencial de Capraria biflora

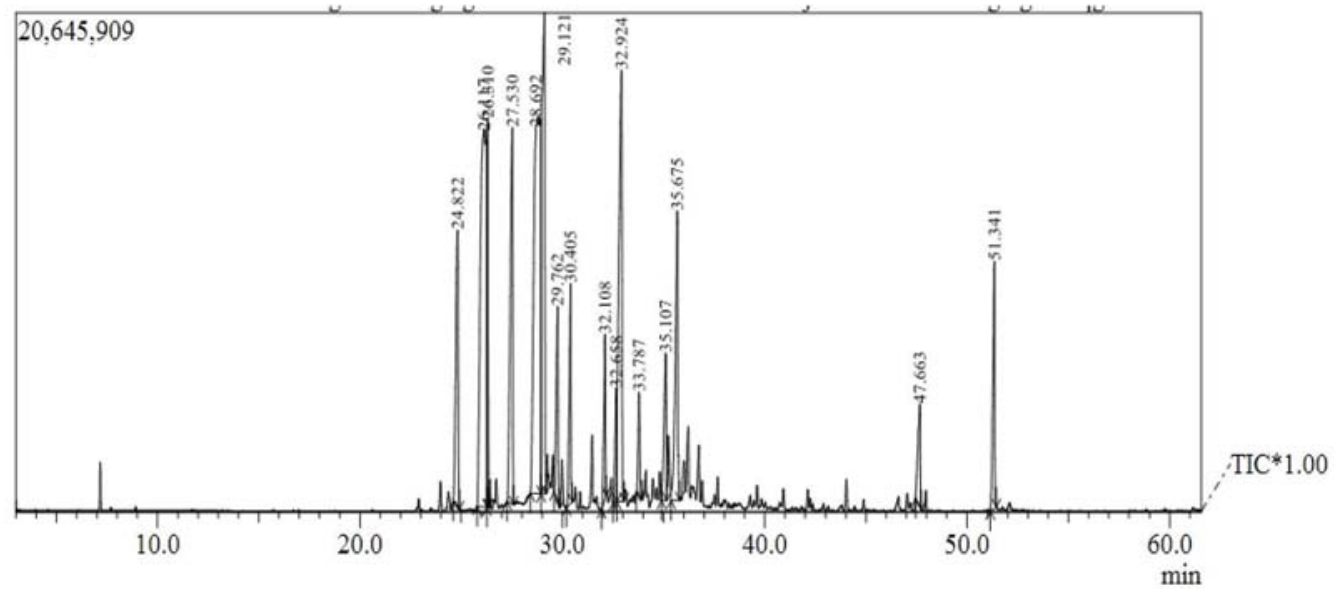

Figura 16S. Cromatograma CG-EM do óleo essencial de Capraria biflora 


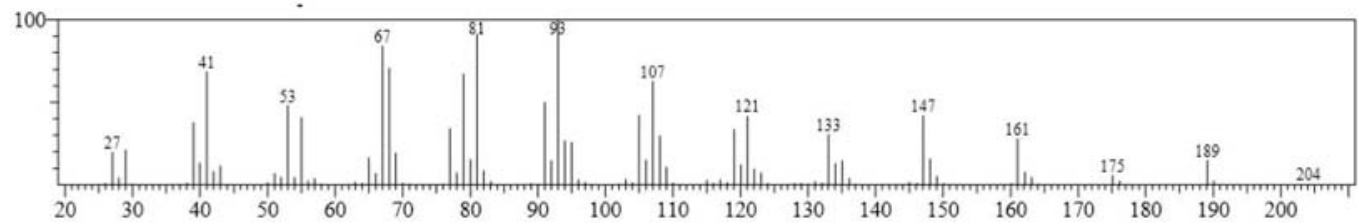

Figura 17S. Espectro de massas do $\beta$-elemeno

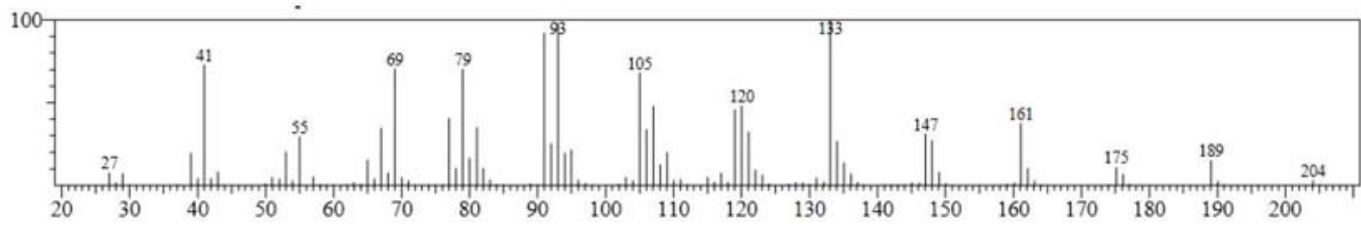

Figura 18S. Espectro de massas do trans-cariofileno

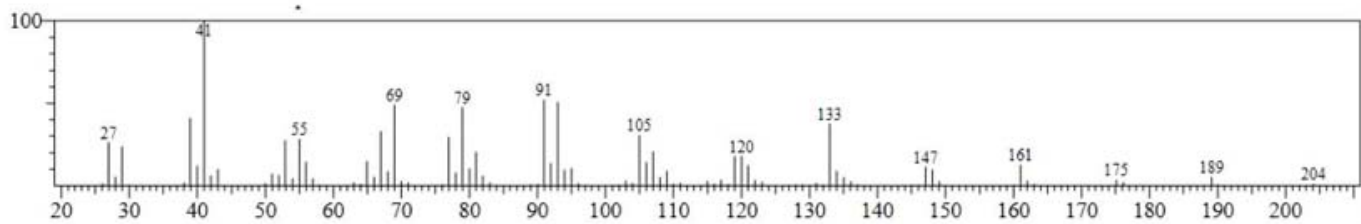

Figura 19S. Espectro de massas do aromadendreno

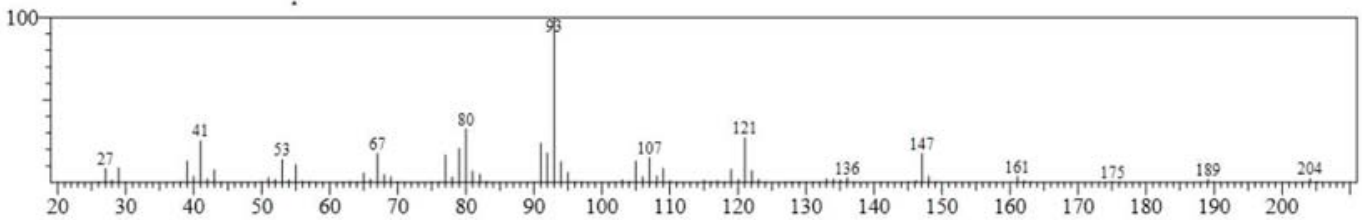

Figura 20S. Espectro de massas do $\alpha$-humuleno

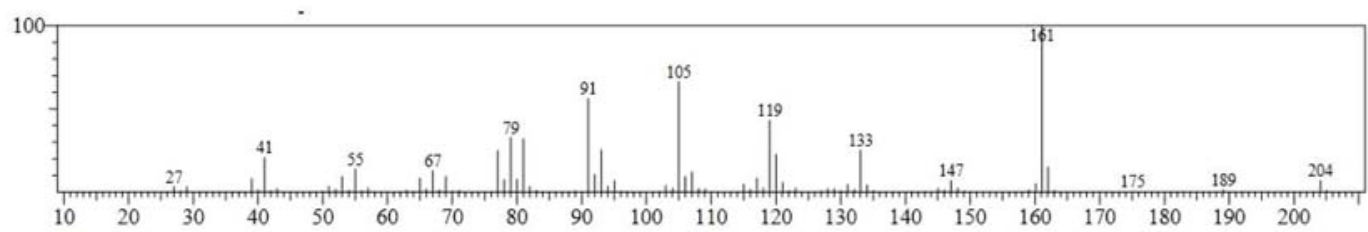

Figura 21S. Espectro de massas do $\gamma$-muuroleno

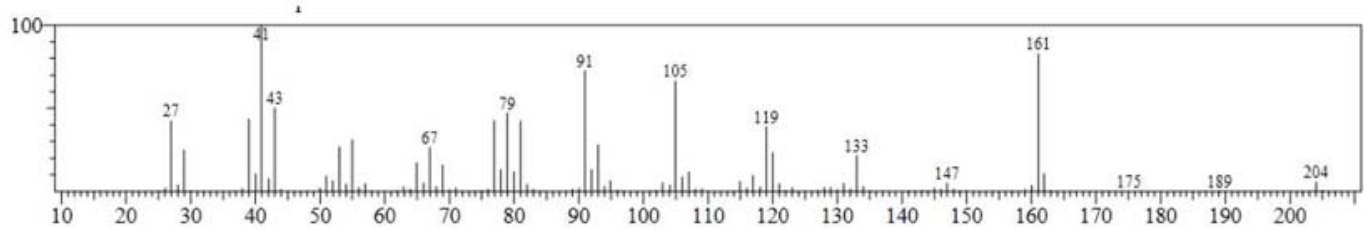

Figura 22S. Espectro de massas do germacreno D

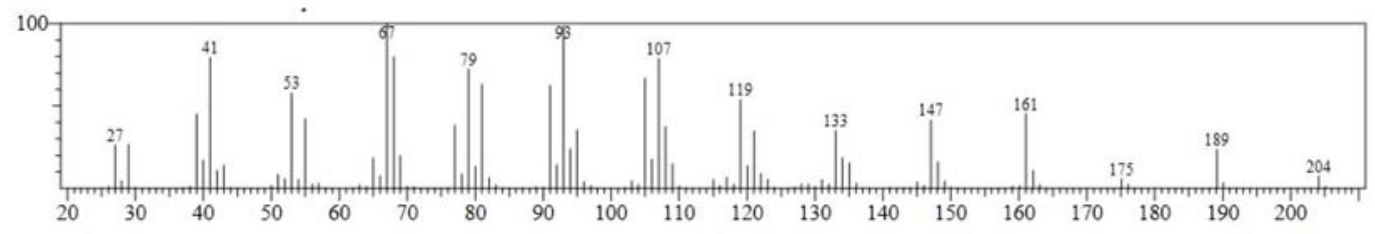

Figura 23S. Espectro de massas do 8-Isopropenil-1,5-dimetil-ciclodeca-1,5-dieno 


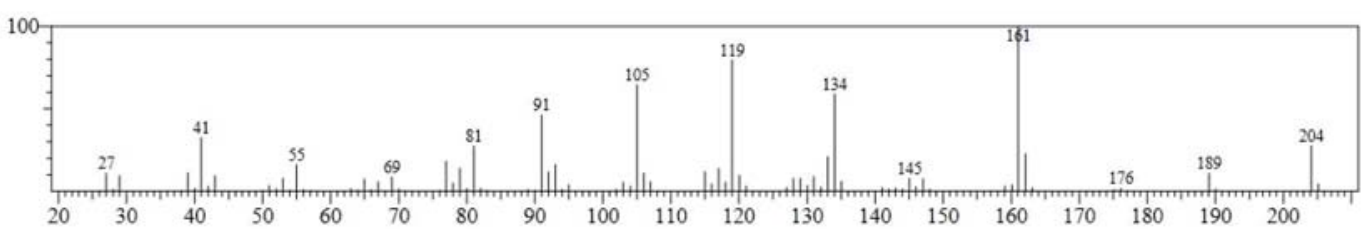

Figura 24S. Espectro de massas do $\delta$-cadineno

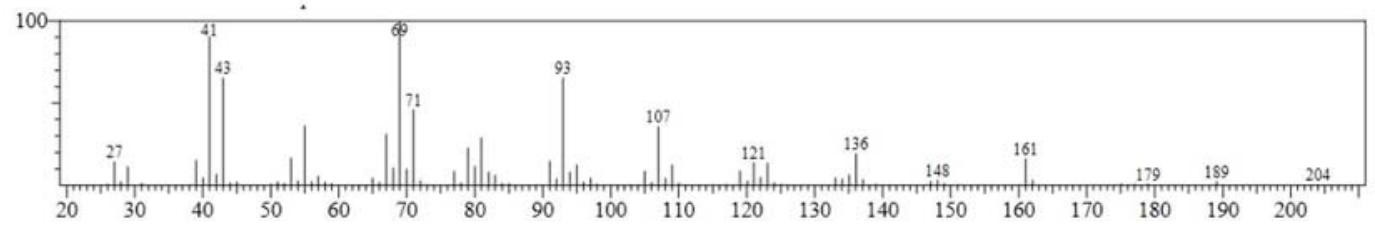

Figura 25S. Espectro de massas do E-nerolidol

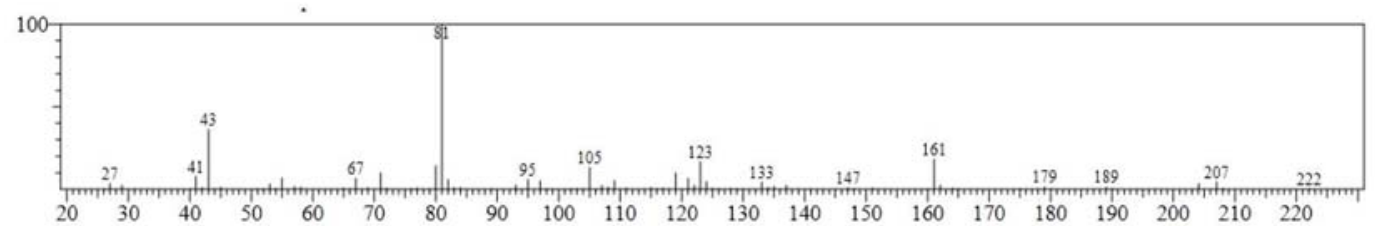

Figura 26S. Espectro de massas do germacren-D-ol

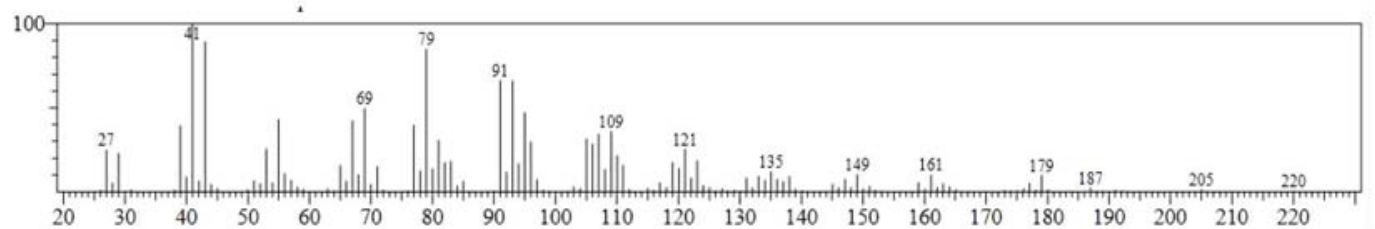

Figura 27S. Espectro de massas do óxido de cariofileno

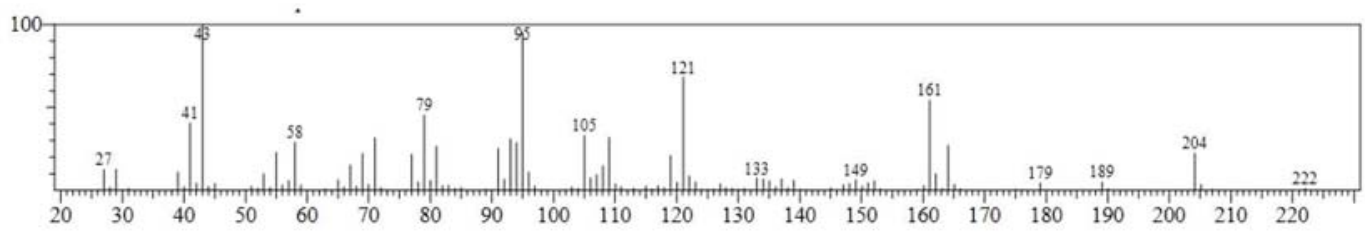

Figura 28S. Espectro de massas do tau-muurolol

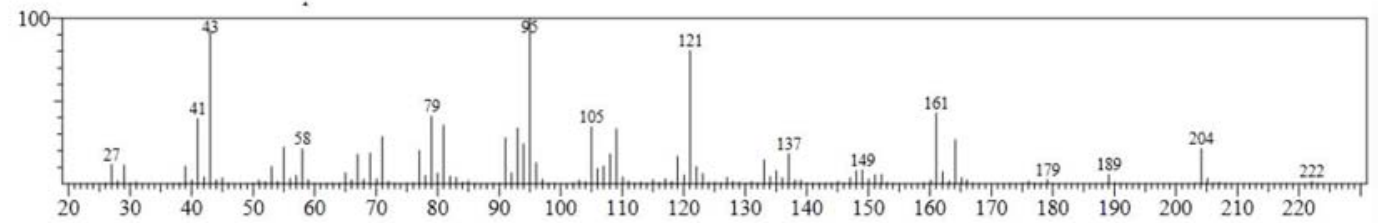

Figura 29S. Espectro de massas do $\alpha$-cadinol

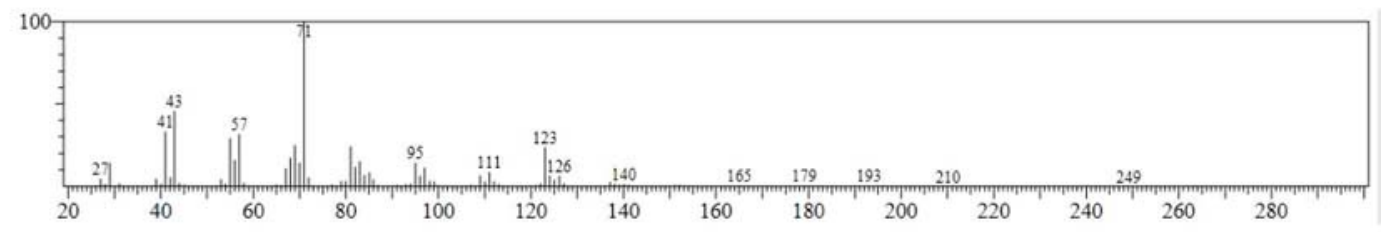

Figura 30S. Espectro de massas do fitol 